\title{
CAUCHY PROBLEMS FOR CERTAIN ISAACS-BELLMAN EQUATIONS AND GAMES OF SURVIVAL
}

\author{
BY \\ ROBERT J. ELLIOTT AND NIGEL J. KALTON
}

\begin{abstract}
Two person zero sum differential games of survival are considered; these terminate as soon as the trajectory enters a given closed set $F$, at which time a cost or payoff is computed. One controller, or player, chooses his control values to make the payoff as large as possible, the other player chooses his controls to make the payoff as small as possible. A strategy is a function telling a player how to choose his control variable and values of the game are introduced in connection with there being a delay before a player adopts a strategy. It is shown that various values of the differential game satisfy dynamic programming identities or inequalities and these results enable one to show that if the value functions are continuous on the boundary of $F$ then they are continuous everywhere. To discuss continuity of the values on the boundary of $F$ certain comparison theorems for the values of the game are established. In particular if there are sub- and super-solutions of a related Isaacs-Bellman equation then these provide upper and lower bounds for the appropriate value function. Thus in discussing value functions of a game of survival one is studying solutions of a Cauchy problem for the Isaacs-Bellman equation and there are interesting analogies with certain techniques of classical potential theory.
\end{abstract}

1. Introduction. The present work is a sequel to our earlier papers on differential games ([1], [2], [3]). As before, a differential game is a dynamical system whose dynamics are described by a family of differential equations, in which there are present two controllers or players $J_{1}$ and $J_{2}$ with directly conflicting interests. In papers [1] and [2] we discussed fixed time games, and in [3] we treated pursuit-evasion games and generalized pursuit-evasion games which end when the trajectory enters a certain set $F$, called the terminal set. The time at which this occurs is called the capture time. When the game ends a real valued quantity called the payoff is computed. For fixed time games the payoff is of the form of an integral of a positive or negative function together with a possibly nonlinear functional on the space of trajectories. In generalized pursuit-evasion games the payoff is the integral up to the capture time of some nonnegative function $h$, whilst for pursuit-evasion games $h$ is identically one so that the payoff is just the time elapsed before capture. During the course of the game the two players can affect the outcome of the game by choosing certain control variables. The greatest value that $J_{1}$ can force is called the lower value of the game, whilst the least value the minimizing player $J_{2}$ can force is called the upper value.

Received by the editors October 13, 1972.

AMS (MOS) subject classifications (1970). Primary 90D25; Secondary 35F30, 31C05.

Key words and phrases. Differential game, dynamical system, dynamic programming, Cauchy problem, potential theory. 
This paper is concerned with games in which the payoff consists of the integral up to the capture time of a function $h$, which may be positive or negative, together with a terminal payoff (i.e. a function $g$ of the final position as the trajectory enters $F$ ). Such games are called games of survival. Because $h$ may change sign it is not a priori clear at any initial point which player should aim to terminate the game by trying to force the trajectory into $F$, so the situation is considerably more complicated than generalized pursuit-evasion games.

In $\$ 2$ differential games of survival and their value functions are defined. New value functions $Q^{+}$and $Q^{-}$are introduced; these naturally occur because of the delays when strategies are pieced together. Dynamic programming results are obtained in $\$ 3$ relating values at any initial position with values at a later position.

Using the results of $\$ 3$ the value functions are shown in $\$ 4$ to be solutions (or, respectively, sub- or super-solutions) of the Isaacs-Bellman equation at points of differentiability.

If there is a $C^{1}$ sub-solution $\theta$ of the Isaacs-Bellman equation satisfying $\theta(t, x) \geq g(t, x)$ on the boundary of $F$, then approximation results proved in $\S 5$ enable one to show $\theta(t, x) \geq Q^{+}(t, x)$.

The boundary $\partial F$ of $F$ is said to be regular (see Definition 6.2) if certain value functions are continuous on $\partial F$. It is shown in $\$ 6$ that if $\partial F$ is regular then, roughly speaking, the value functions $Q^{+}$and $V^{+}$are then continuous everywhere. The problem of determining sufficient conditions for the regularity of $\partial F$ is, therefore, of some interest and in $\$ 7$, using the estimates of $\$ 5$, it is shown that $\partial F$ is regular if there exist sub- and super-solutions of the Isaacs-Bellman equation or a related equation.

Some methods used in $\$ 7$ are interesting adaptations of techniques used by Perron [10] in discussing the regularity of boundary problems for the Laplace operator. Finally, in $\$ 8$, all the upper and lower values are shown to be equal if $\partial F$ is regular and the Isaacs condition is satisfied.

Some of our results are extensions of those of Friedman [4] for survival games, but all the techniques and the emphasis of this paper are rather different. Indeed, although the work has its origins in differential games of survival, as the above summary indicates it is really more concerned with functions related to solutions, and sub- and super-solutions, of Cauchy problems for the Isaacs-Bellman equation. For example, the results of $\$ 4$ show that if the value function of the differential game is Lipschitz continuous then it is a generalized solution of this Cauchy problem. As the reference to Perron above indicates this is reminiscent of techniques of probabilistic potential theory where solutions to certain parabolic equations are constructed using an associated random process. Using the methods above one can obtain results concerning solutions to Cauchy problems for a whole family of nonlinear partial differential equations by constructing related differential games of survival, but this is something we discuss in another paper. 
We wish to thank A. Friedman for finding an error in the original draft of this paper and for simplifying the proof of Lemma 4.3 by observing that the map of Lemma 4.2 is in fact a strategy.

2. Differential games and values. Let $Y$ and $Z$ be compact metric spaces; we consider the differential equation

$$
d x / d t=\dot{x}(t)=f(t, x, y, z)
$$

where $x \in R^{m}$ and $t$, denoting time, belongs to $\left[t_{0}, \infty\right] . f: R \times R^{m} \times Y \times Z \rightarrow R^{m}$ is continuous and satisfies a Lipschitz condition in $x$ :

$$
\left\|f\left(t, x_{1}, y, z\right)-f\left(t, x_{2}, y, z\right)\right\| \leq K\left\|x_{1}-x_{2}\right\|,
$$

where $x_{1}, x_{2} \in R^{m}$. We shall consider a differential game of survival $G\left(t_{0}, x_{0}\right)$ associated with (1) and the initial conditions

$$
x\left(t_{0}\right)=x_{0},
$$

terminal set $F \subset R \times R^{m}$, where $F$ is closed and $F \supset[T, \infty] \times R^{m}$ for some fixed $T$, and payoff

$$
P(y, z)=g\left(t_{F}, x\left(t_{F}\right)\right)+\int_{t_{0}}^{t_{F}} h(t, x(t), y(t), z(t)) d t .
$$

Here $t_{F}$ is the first time of entry of the trajectory $x(t)$ into the terminal set, $h: R \times R^{m} \times Y \times Z \rightarrow R$ is continuous, and $g: R \times R^{m} \rightarrow R$ is continuous. The player $J_{1}$ controlling $y \in Y$ is trying to maximize $P$ whilst $J_{2}$ is trying to minimize $P$.

A map $y:\left[t_{0}, \infty\right] \rightarrow Y$ is measurable if for every continuous real valued function $\psi$ on $Y, \psi \circ y$ is Lebesgue measurable. Denote by $\mathcal{M}_{1}\left(t_{0}\right)$ the space of such measurable functions, where two functions equal almost everywhere are identified. Similarly $\mathcal{M}_{2}\left(t_{0}\right)$ denotes the space of measurable maps $\left[t_{0}, \infty\right] \rightarrow Z$. For $s \geq 0$ we define an s-delay strategy $\alpha$ for $J_{1}$ as a map $\alpha$ : $\delta M_{2}\left(t_{0}\right) \rightarrow \propto M_{1}\left(t_{0}\right)$ such that whenever

$$
z_{1}(t)=z_{2}(t) \text { a.e. } t_{0} \leq t \leq t_{1}
$$

then $\left(\alpha z_{1}\right)(t)=\left(\alpha z_{2}\right)(t)$ a.e. $t_{0} \leq t \leq t_{1}+s$. (Note that the values of $y=\alpha(z)$ in $\left[t_{0}, t_{0}+s\right)$ are independent of the function $z(s)$.) The set of $s$-delay strategies is denoted by $\Gamma_{t_{0}}(s)$. We define $s$-delay strategies for $J_{2}$ similarly and denote the set by $\Delta_{t_{0}}(s)$.

The value $u(\alpha)$ of $\alpha \in \Gamma_{t_{0}}(0)$ is defined as

$$
u(\alpha)=\inf \left[P(\alpha z, z) ; z \in \mathcal{O M}_{2}\left(t_{0}\right)\right],
$$

and then 


$$
U\left(t_{0}, x_{0}\right)=\sup \left[u(\alpha) ; \alpha \in \Gamma_{t_{0}}(0)\right]
$$

Similarly, for $\beta \in \Delta_{t_{0}}(0)$,

$$
v(\beta)=\sup \left[P(y, \beta y) ; y \in M_{1}\left(t_{0}\right)\right]
$$

and

$$
V\left(t_{0}, x_{0}\right)=\inf \left[v(\beta) ; \beta \in \Delta_{t_{0}}(0)\right]
$$

The upper and lower values $\mathrm{V}^{+}$and $\mathrm{V}^{-}$are defined by

$$
\begin{aligned}
& V^{+}\left(t_{0}, x_{0}\right)=\inf \left[u(\beta) ; \beta \in \bigcup_{s>0} \Delta_{t_{0}}(s)\right], \\
& V^{-}\left(t_{0}, x_{0}\right)=\sup \left[u(\alpha) ; \alpha \in \cup_{s>0} \Gamma_{t_{0}}(s)\right] ;
\end{aligned}
$$

and in general we have (see [3]) $V^{-} \leq U, V \leq V^{+}$.

For games of survival it is necessary to introduce another concept of value. First we define the $s$-perturbed value of a strategy; this is the value of a strategy when it is adopted only after a time $s$ has elapsed. Thus in the initial interval $\left[t_{0}, t_{0}+s\right)$ the player is assumed to adopt any possible control function, conceivably the worst available. We say for two strategies $\alpha, \alpha^{\prime} \in \Gamma_{t_{0}}(0)$, that $\alpha^{\prime}$ $\in \Gamma_{t_{0}}^{s}(\alpha)$ if for any $z \in M_{2}\left(t_{0}\right)$

$$
\left(\alpha^{\prime} z\right)(t)=(\alpha z)(t) \text { a.e. } t_{0}+s \leq t<\infty .
$$

Similarly we say for $\beta, \beta^{\prime} \in \Delta_{t_{0}}(0)$ that $\beta^{\prime} \in \Delta_{t_{0}}^{s}(\beta)$ if for any $y \in M_{1}\left(t_{0}\right)$

$$
\left(\beta^{\prime} y\right)(t)=(\beta y)(t) \text { a.e. } t_{0}+s \leq t<\infty .
$$

The s-perturbed value of $\alpha \in \Gamma_{t_{0}}(0)$ is then defined as $u_{s}(\alpha)=\inf \left[u\left(\alpha^{\prime}\right) ; \alpha^{\prime}\right.$ $\left.\in \Gamma_{t_{0}}^{s}(\alpha)\right]$ and the s-perturbed value of $\beta \in \Delta_{t_{0}}(0)$ is defined as $v_{s}(\beta)$ $=\sup \left[v\left(\beta^{\prime}\right) ; \beta^{\prime} \in \Delta_{t_{0}}^{s}(\beta)\right]$.

We write $\mathcal{M}_{1}^{s}\left[t_{0}\right]$ for the space of measurable functions $y:\left[t_{0}, t_{0}+s\right] \rightarrow Y$ and also define $\Gamma_{t_{0}}[s \mid y]$ as the set of strategies $\alpha \in \Gamma_{t_{0}}(s)$ such that $\alpha z(t)=y(t)$ a.e.

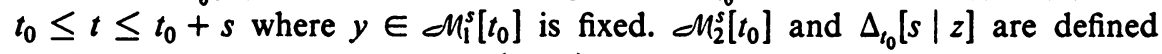
similarly. We define the value $Q_{s}^{-}\left(t_{0}, x_{0}\right)$ by

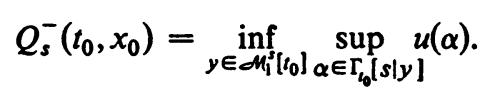

Similarly we define

$$
Q_{s}^{+}\left(t_{0}, x_{0}\right)=\sup _{z \in \mathcal{N}_{2}^{s}\left[t_{0}\right]} \inf _{\left.\beta \in \Delta_{0}[s] z\right]} v(\beta) .
$$

Then clearly we have the relations

$$
Q_{s}^{-}\left(t_{0}, x_{0}\right) \geq \sup _{\alpha \in \Gamma_{t_{0}}(s)} u_{s}(\alpha) \text { and } Q_{s}^{+}\left(t_{0}, x_{0}\right) \leq \inf _{\beta \in \Delta_{t_{0}}(s)} v_{s}(\beta) .
$$


Next we define

$$
\begin{aligned}
& Q^{+}\left(t_{0}, x_{0}\right)=\lim _{s \rightarrow 0} Q_{s}^{+}\left(t_{0}, x_{0}\right)=\inf _{s>0} Q_{s}^{+}\left(t_{0}, x_{0}\right), \\
& Q^{-}\left(t_{0}, x_{0}\right)=\lim _{s \rightarrow 0} Q_{s}^{-}\left(t_{0}, x_{0}\right)=\sup _{s>0} Q_{s}^{-}\left(t_{0}, x_{0}\right) .
\end{aligned}
$$

The quantities $Q^{+}$and $Q^{-}$are alternative definitions of upper and lower value, in place of $V^{+}$and $V^{-}$. However, if $Q^{+}=Q^{-}$then we can certainly assert that $V^{+}=V^{-}$.

Lemma 2.1. $Q^{-}\left(t_{0}, x_{0}\right) \leq V^{-}\left(t_{0}, x_{0}\right)$ and $V^{+}\left(t_{0}, x_{0}\right) \leq Q^{+}\left(t_{0}, x_{0}\right)$.

Proof. This is immediate from the definitions, for clearly

$$
Q_{s}^{-}\left(t_{0}, x_{0}\right)=\inf _{y \in \mathcal{M} M_{i}^{i}\left[t_{0}\right]} \sup _{\alpha \in \Gamma_{0}[s \mid y]} u(\alpha) \leq V^{-}\left(t_{0}, x_{0}\right) \text { for } s>0
$$

and so $Q^{-}\left(t_{0}, x_{0}\right) \leq V^{-}\left(t_{0}, x_{0}\right)$. The other inequality is similarly proved.

Lemma 2.2. If $G$ is a fixed time game (i.e. $\left.F=[T, \infty] \times R^{m}\right)$ then $Q^{+}\left(t_{0}, x_{0}\right)$ $=V^{+}\left(t_{0}, x_{0}\right)$ and $Q^{-}\left(t_{0}, x_{0}\right)=V^{-}\left(t_{0}, x_{0}\right)$.

Proof. We sketch then details only. If $\alpha \in \Gamma_{t_{0}}(0)$ then $\left|P\left(\alpha^{\prime} z, z\right)-P(\alpha z, z)\right|$ $=O(s)$ uniformly in $z \in M_{2}\left(t_{0}\right), \alpha^{\prime} \in \Gamma_{t_{0}}^{s}(\alpha)$ and $\alpha \in \Gamma_{t_{0}}(0)$. Therefore $\mid u\left(\alpha^{\prime}\right)$ $-u(\alpha) \mid=O(s)$ so $\left|u(\alpha)-u_{s}(\alpha)\right|=O(s)$ uniformly in $\alpha \in \Gamma_{t_{0}}(0)$. In particular, for $\alpha \in \Gamma_{t_{0}}(s)$, since $Q_{s}^{-}\left(t_{0}, x_{0}\right) \geq \sup _{\alpha \in \Gamma_{0}(s)} u_{s}(\alpha), u(\alpha) \leq Q_{s}^{-}\left(t_{0}, x_{0}\right)+O(s)$. Therefore $V^{-}\left(t_{0}, x_{0}\right) \leq Q^{-}\left(t_{0}, x_{0}\right)$ and so $V^{-0}\left(t_{0}, x_{0}\right)=Q^{-}\left(t_{0}, x_{0}\right)$ by Lemma 2.1.

The following example shows that in games of survival the situation is more complicated. We consider only a simple game in which $J_{1}$ (the maximizer) appears only in a trivial role, i.e. he has no effect on the course of the game. In this case the game reduces to an optimal control problem for $J_{2}$, the minimizer.

Example 2.3. Let $Z \subset R^{2}$ consist of all points $z=\left(\xi_{1}, \xi_{2}\right)$ such that $\xi_{1} \geq 0$ and $\xi_{1}^{2}+\xi_{2}^{2} \leq 1$. We consider a game played in $R^{2}$ with dynamics

$$
d x / d t=z \in R^{2},
$$

$t_{0}=0$, initial conditions $x_{0}=x(0)=\left(0, \frac{1}{4}\right)$ and terminal set $F=\left\{\left(t, \xi_{1}, \xi_{2}\right) ; \xi_{1}\right.$ $\left.\leq 0, \xi_{2} \leq 0\right\} \cup\left\{\left(t, \xi_{1}, \xi_{2}\right) ; t \geq 1\right\}$. The payoff is

$$
P=\int_{0}^{t_{F}} 1 d t=t_{F}
$$

It is clear that $J_{2}$ 's optimal strategy is to adopt $z(t) \equiv(0,-1)$ and in this case the payoff is given by $t_{F}=\frac{1}{4}$, so that $V^{+}\left(t_{0}, x_{0}\right)=\frac{1}{4}$. However, if $s>0$ and $J_{2}$ has no control over his choice of $z(t)$ for $0 \leq t \leq s$, then the trajectory may move to the point $\left(s, \frac{1}{4}\right)$, and then no possible choice of $z(t)$ results in a payoff of less than one. Hence $Q^{+}\left(t_{0}, x_{0}\right)=1$.

3. Dynamic programming. We now consider a very general type of differential game formed from the original game $G\left(t_{0}, x_{0}\right)$. Let $\theta$ be any function (not 
necessarily continuous) defined on $R \times R^{m}$ and let $\tau$ be any map $\tau: \delta M_{1}\left(t_{0}\right)$ $\times \propto M_{2}\left(t_{0}\right) \rightarrow\left[t_{0}, \infty\right)$ which prescribes a stopping time $\tau(y, z)$ corresponding to any pair of control functions. We define a game $G_{\tau}\left(t_{0}, x_{0} ; \theta\right)$ as the game with initial condition (3), dynamics (1) and payoff:

$$
P_{r, \theta}(y, z)=\theta(\tau(y, z), x(\tau(y, z)))+\int_{t_{0}}^{\tau(y, z)} h(t, x(t), y(t), z(t)) d t .
$$

For $\alpha \in \Gamma_{t_{0}}(0)$ we define

$$
u_{\tau, \theta}(\alpha)=\inf \left[P_{r, \theta}(\alpha z, z) ; z \in \quad M_{2}\left(t_{0}\right)\right]
$$

and

$$
u_{s, r, \theta}(\alpha)=\inf \left[u_{\tau, \theta}\left(\alpha^{\prime}\right) ; \alpha^{\prime} \in \Gamma_{t_{0}}^{s}(\alpha)\right]
$$

For $\beta \in \Delta_{t_{0}}(0)$ similarly

$$
\begin{aligned}
v_{\tau, \theta}(\beta) & =\sup \left[P_{r, \theta}(y, \beta y) ; y \in M_{1}\left(t_{0}\right)\right], \\
v_{s, \tau, \theta}(\beta) & =\sup \left[v_{\tau, \theta}\left(\beta^{\prime}\right) ; \beta^{\prime} \in \Delta_{t_{0}}^{s}(\beta)\right] .
\end{aligned}
$$

Then, by exact analogy with the game $G\left(t_{0}, x_{0}\right)$,

$$
\begin{aligned}
& U_{\tau}\left(t_{0}, x_{0} ; \theta\right)=\sup \left[u_{\tau, \theta}(\alpha) ; \alpha \in \Gamma_{t_{0}}(0)\right], \\
& V_{\tau}\left(t_{0}, x_{0} ; \theta\right)=\inf \left[v_{\tau, \theta}(\beta) ; \beta \in \Delta_{t_{0}}(0)\right] \text {, } \\
& V_{\tau}^{-}\left(t_{0}, x_{0} ; \theta\right)=\sup \left[u_{\tau, \theta}(\alpha) ; \alpha \in \bigcup_{s>0} \Gamma_{t_{0}}(s)\right] \text {, } \\
& V_{\tau}^{+}\left(t_{0}, x_{0} ; \theta\right)=\inf \left[v_{\tau, \theta}(\beta) ; \beta \in \bigcup_{s>0} \Delta_{t_{0}}(s)\right] \text {, } \\
& Q_{s, \tau}^{-}\left(t_{0}, x_{0} ; \theta\right)=\inf _{y \in \mathcal{L} \mathcal{M}_{[}^{j}\left[t_{0}\right]} \sup \left[u_{\tau, \theta}(\alpha) ; \alpha \in \Gamma_{t_{0}}[s \mid y]\right] \text {, } \\
& Q_{s, \tau}^{+}\left(t_{0}, x_{0} ; \theta\right)=\sup _{\left.z \in \mathcal{M}_{2}^{i} t_{0}\right]} \inf \left[v_{\tau, \theta}(\beta) ; \beta \in \Delta_{t_{0}}[s \mid z]\right] \text {, } \\
& Q_{\tau}^{-}\left(t_{0}, x_{0} ; \theta\right)=\sup _{s>0} Q_{s, \tau}^{-}\left(t_{0}, x_{0} ; \theta\right), \\
& Q_{\tau}^{+}\left(t_{0}, x_{0} ; \theta\right)=\inf _{s>0} Q_{s, \tau}^{+}\left(t_{0}, x_{0} ; \theta\right) .
\end{aligned}
$$

As usual we have the relationships

$$
\begin{aligned}
& Q_{\tau}^{-}\left(t_{0}, x_{0} ; \theta\right) \leq V_{\tau}^{-}\left(t_{0}, x_{0} ; \theta\right) \leq U_{\tau}\left(t_{0}, x_{0} ; \theta\right), \\
& Q_{\tau}^{+}\left(t_{0}, x_{0} ; \theta\right) \geq V_{\tau}^{+}\left(t_{0}, x_{0} ; \theta\right) \geq V_{\tau}\left(t_{0}, x_{0} ; \theta\right) .
\end{aligned}
$$

Furthermore, since any $\alpha \in \Gamma_{t_{0}}(0)$ and $\beta \in \Delta_{t_{0}}(s)$ produce a unique control pair $y \in \delta M_{1}\left(t_{0}\right), z \in \delta M_{2}\left(t_{0}\right)$, such that $\beta y=z$ and $\alpha z=y$, we have

$$
U_{\tau}\left(t_{0}, x_{0} ; \theta\right) \leq V_{\tau}^{+}\left(t_{0}, x_{0} ; \theta\right)
$$


and similarly

$$
V_{\tau}\left(t_{0}, x_{0} ; \theta\right) \geq V_{\tau}^{-}\left(t_{0}, x_{0} ; \theta\right) .
$$

We now use games $G_{\tau}\left(t_{0}, x_{0} ; \theta\right)$ to derive several "dynamic programming" results relating the values of $G\left(t_{0}, x_{0}\right)$ to those of $G_{\tau}\left(t_{0}, x_{0} ; \theta\right)$ for various $\theta$. Note that $G\left(t_{0}, x_{0}\right)$ is just the game $G_{t_{F}}\left(t_{0}, x_{0} ; g\right)$; we shall consider $\tau$ such that $\tau(y, z) \leq t_{F}(y, z)$ for all $y, z . \tau$ is said to be nonanticipating if whenever

$$
\begin{aligned}
& y^{\prime}(t)=y(t) \quad \text { a.e. } t_{0} \leq t \leq \tau(y, z) \\
& z^{\prime}(t)=z(t) \quad \text { a.e. } t_{0} \leq t \leq \tau(y, z)
\end{aligned}
$$

then $\tau\left(y^{\prime}, z^{\prime}\right)=\tau(y, z)$.

Note that $t_{F}$ is nonanticipating.

Theorem 3.1. Suppose $\tau$ is nonanticipatory and $\tau \leq t_{F}$. Then

$$
\begin{aligned}
& U\left(t_{0}, x_{0}\right)=U_{\tau}\left(t_{0}, x_{0} ; U\right), \\
& V\left(t_{0}, x_{0}\right)=V_{\tau}\left(t_{0}, x_{0} ; V\right) .
\end{aligned}
$$

Proof. Only the former identity will be proved; the second follows by symmetry. For $(t, x) \in R^{m+1}$ and $\varepsilon>0$ there is an $\alpha=\alpha(t, x) \in \Gamma_{t}(0)$ such that $u(\alpha) \geq U(t, x)-(\varepsilon / 2)$. There is also an $\alpha_{0} \in \Gamma_{t_{0}}(0)$ such that $u_{\tau, U}\left(\alpha_{0}\right) \geq U_{\tau}\left(t_{0}\right.$, $\left.x_{0} ; U\right)-(\varepsilon / 2)$. Now define $\alpha^{\prime} \in \Gamma_{t_{0}}(0)$ as follows: For $z \in \mathcal{O} M_{2}\left(t_{0}\right)$ let $\sigma(z)$ $=\tau\left(\alpha_{0} z, z\right)$ and define $\left(\alpha^{\prime} z\right)(t)=\left(\alpha_{0} z\right)(t), t_{0} \leq t \leq \sigma(z)$,

$$
\left(\alpha^{\prime} z\right)(t)=\alpha(\sigma(z), x(\sigma(z))) z(t), \quad \sigma(z)<t<\infty,
$$

where $x(t)$ is the trajectory corresponding to $\left(\alpha_{0} z, z\right)$. The second equality above is interpreted by restricting $z$ to an element of $\mathcal{M}_{2}(\sigma(z))$. It is clear that $\alpha^{\prime} \in \Gamma_{t_{0}}(0)$ and furthermore

$$
\begin{aligned}
P\left(\alpha^{\prime} z, z\right) & =g\left(t_{F}, x\left(t_{F}\right)\right)+\int_{t_{0}}^{t_{F}} h d t \\
& =\int_{t_{0}}^{\sigma(z)} h d t+\left\{\int_{\alpha(z)}^{t_{F}} h d t+g\left(t_{F}, x\left(t_{F}\right)\right)\right\} \\
& \geq \int_{t_{0}}^{\alpha(z)} h d t+U(\sigma(z), x(\sigma(z)))-(\varepsilon / 2) \\
& \geq P_{\tau, U}\left(\alpha_{0} z, z\right)-(\varepsilon / 2) \\
& \geq U_{\tau}\left(t_{0}, x_{0} ; U\right)-\varepsilon
\end{aligned}
$$

so that $U\left(t_{0}, x_{0}\right) \geq U_{\tau}\left(t_{0}, x_{0} ; U\right)$. Conversely, given $\alpha_{0} \in \Gamma_{t_{0}}(0)$ then there exists a control $z \in M_{2}\left(t_{0}\right)$ with 


$$
\int_{t_{0}}^{\sigma(z)} h d t+U(\sigma(z), x(\sigma(z))) \leq U_{\tau}\left(t_{0}, x_{0} ; U\right)+(\varepsilon / 2)
$$

for the trajectory $x(t)$ corresponding to $\left(\alpha_{0} z, z\right)$. Now define $\bar{\alpha} \in \Gamma_{\sigma(z)}(0)$ by $\bar{\alpha} \omega(t)=\alpha_{0} \bar{\omega}(t), \sigma(z) \leq t<\infty$, where

$$
\begin{aligned}
\bar{\omega}(t) & =z(t), & t_{0} \leq t<\sigma(z), \\
& =\omega(t), & \sigma(z) \leq t<\infty .
\end{aligned}
$$

Then in $G(\sigma(z), x(\sigma(z))): u(\bar{\alpha}) \leq U(\sigma(z), x(\sigma(z)))$ and so there is a control function $\omega_{0} \in M_{2}(\sigma(z))$ with

$$
P\left(\bar{\alpha}_{0} \omega_{0}, \omega_{0}\right) \leq U(\sigma(z), x(\sigma(z)))+(\varepsilon / 2) .
$$

If we now define

$$
\begin{aligned}
\omega_{1}(t) & =z(t), & & t_{0} \leq t<\sigma(z), \\
& =\omega_{0}(t), & & \sigma(z) \leq t<\infty
\end{aligned}
$$

then we have

$$
\begin{aligned}
P\left(\alpha_{0} \omega_{1}, \omega_{1}\right) & =P\left(\bar{\alpha} \omega_{0}, \omega_{0}\right)+\int_{t_{0}}^{o(z)} h d t \\
& \leq \int_{t_{0}}^{o(z)} h d t+U(\sigma(z), x(\sigma(z)))+(\varepsilon / 2) \\
& \leq U_{\tau}\left(t_{0}, x_{0} ; U\right)+\varepsilon
\end{aligned}
$$

so that $U\left(t_{0}, x_{0}\right) \leq U_{\tau}\left(t_{0}, x_{0} ; U\right)$.

We now consider similar results for $V^{+}$and $V^{-}$. However, in this case delay strategies do not fit together as neatly as ordinary strategies and so we only obtain inequalities in place of the equalities of Theorem 3.1.

Theorem 3.2. Suppose $\tau$ is nonanticipating and $\tau \leq t_{F}$. Then

$$
\begin{aligned}
& V^{+}\left(t_{0}, x_{0}\right) \geq V_{\tau}^{+}\left(t_{0}, x_{0} ; V^{+}\right), \\
& V^{-}\left(t_{0}, x_{0}\right) \leq V_{\tau}^{-}\left(t_{0}, x_{0} ; V^{-}\right) .
\end{aligned}
$$

Proof. We prove only the latter inequality. Suppose $\alpha \in \Gamma_{t_{0}}(s)$ for some $s>0$ and $z \in M_{2}\left(t_{\sigma}\right)$. Again write $\sigma z=\sigma(z)=\tau(\alpha z, z)$ and consider $G(\sigma z, x(\sigma z))$ where $x(t)$ is the trajectory corresponding to $(\alpha z, z)$. For $\omega \in M_{2}(\sigma z)$ define $\bar{\omega} \in M_{2}\left(t_{0}\right)$ by

$$
\begin{array}{ll}
\bar{\omega}(t)=z(t), & t_{0} \leq t<\sigma(z), \\
\bar{\omega}(t)=\omega(t), & \sigma(z) \leq t<\infty,
\end{array}
$$

and $\bar{\alpha} \in \Gamma_{o z}(s)$ by 


$$
\bar{\alpha} \omega(t)=\alpha \bar{\omega}(t), \quad \sigma(z) \leq t<\infty .
$$

Note that $\bar{\alpha}$ depends on $z(t), t_{0} \leq t<\sigma z$, and $\bar{\alpha} \omega$ is independent of $\omega$ in $[\sigma z, \sigma z+s)$. In $G(\sigma z, x(\sigma z)), u(\bar{\alpha}) \leq V^{-}(\sigma z, x(\sigma z))$ and so there exists $\omega$ $\in M_{2}(\sigma z)$ such that $P(\bar{\alpha} \omega, \omega) \leq V^{-}(\sigma z, x(\sigma z))+\varepsilon$. Therefore in $G\left(t_{0}, x_{0}\right)$

$$
\begin{aligned}
P(\alpha \bar{\omega}, \bar{\omega}) & \leq \int_{\tau_{0}}^{\sigma(z)} h d t+V^{-}(\sigma z, x(\sigma z))+\varepsilon \\
& \leq P_{\tau, V^{-}}(\alpha \bar{\omega}, \bar{\omega})+\varepsilon \\
& =P_{\tau, V^{-}}(\alpha z, z)+\varepsilon .
\end{aligned}
$$

Hence $u(\alpha) \leq P_{\tau, V^{-}}(\alpha z, z)+\varepsilon$ and so $u(\alpha) \leq u_{\tau, V^{-}}(\alpha)+\varepsilon$ and (15) follows.

Finally we consider $Q^{+}$and $Q^{-}$; in this case we first have to treat $Q_{s}^{+}$and $Q_{s}^{-}$.

Theorem 3.3. Suppose $\tau$ is nonanticipating and $s \leq \tau \leq t_{F}$. Then

$$
\begin{aligned}
& Q_{s}^{+}\left(t_{0}, x_{0}\right) \leq Q_{s, \tau}^{+}\left(t_{0}, x_{0} ; Q_{s}^{+}\right), \\
& Q_{s}^{-}\left(t_{0}, x_{0}\right) \geq Q_{s, \tau}^{-}\left(t_{0}, x_{0} ; Q_{s}^{-}\right) .
\end{aligned}
$$

Proof. We prove only (17). For $\varepsilon>0,(t, x) \in R^{m+1}$ and $y \in \mathcal{M}\left(t_{0}\right)$ there exists $\alpha=\alpha(t, x, y) \in \Gamma_{t}[s \mid y]$ such that, in $G(t, x), u(\alpha) \geq Q_{s}^{-}(t, x)-\varepsilon$.

Now suppose $\alpha_{0} \in \Gamma_{t}(s)$. For any $z \in \mathcal{M}_{2}\left(t_{0}\right)$ define $\sigma z=\tau\left(\alpha_{0} z, z\right)$ and suppose $x(t)$ is the trajectory induced by $\left(\alpha_{0} z, z\right), \bar{\alpha} \in \Gamma_{t_{0}}(s)$ is then defined by

$$
\begin{array}{ll}
\bar{\alpha} z(t)=\alpha_{0} z(t), & t_{0} \leq t<\sigma z+s, \\
\bar{\alpha} z(t)=\alpha(\sigma z, x(\sigma z), \bar{y}) z(t), & \sigma z+s \leq t<\infty,
\end{array}
$$

where $\bar{y}(t)=\alpha_{0} z(t), \sigma z \leq t \leq \sigma z+s$. It is easy to check that $\bar{\alpha} \in \Gamma_{t_{0}}(s)$. For example, suppose $z_{1}(t)=z_{2}(t)$ a.e. $t_{0} \leq t \leq \sigma z+s_{1}$, where $0<s_{1} \leq s$. Then $\sigma z_{1}=\sigma z_{2}$ and $\alpha_{0} z_{1}(t)=\alpha_{0} z_{2}(t)$ a.e. $t_{0} \leq t \leq \sigma z+s_{1}+s$, and in particular

$$
\bar{y}_{1}(t)=\bar{y}_{2}(t) \text { a.e. } \sigma z \leq t \leq \sigma z+s .
$$

Hence $\alpha\left(\sigma z_{1}, x\left(\sigma z_{1}\right), \bar{y}_{1}\right)=\alpha\left(\sigma z_{2}, x\left(\sigma z_{2}\right), \bar{y}_{2}\right)$ and so

$$
\bar{\alpha} z_{1}(t)=\bar{\alpha} z_{2}(t) \text { a.e. } t_{0} \leq t \leq \sigma z+s+s_{1} .
$$

Now $P(\bar{\alpha} z, z)=\int_{t_{0}}^{\sigma z} h d t+P^{*}\left(\alpha^{\prime} z, z\right)$ where $P^{*}$ denotes the payoff in $G(\sigma z, x(\sigma z))$ and $\alpha^{\prime}=\alpha(\sigma z, x(\sigma z), \bar{y}) \in \Gamma_{\sigma z}(s)$. Thus

$$
\begin{aligned}
P(\bar{\alpha} z, z) & \geq \int_{t_{0}}^{\sigma z} h d t+u\left(\alpha^{\prime}\right) \\
& \geq \int_{t_{0}}^{\sigma z} h d t+u(\alpha(\sigma z, x(\sigma z))) \geq \int_{t_{0}}^{\sigma z} h d t+Q_{s}^{-}(\sigma z, x(\sigma z))-\varepsilon \\
& =P_{\tau, Q_{s}}(\bar{\alpha} z, z)-\varepsilon=P_{\tau, Q_{s}}\left(\alpha_{0} z, z\right)-\varepsilon .
\end{aligned}
$$


Therefore $u(\bar{\alpha}) \geq u_{\tau, Q_{s}^{-}}\left(\alpha_{0}\right)-\varepsilon$. Suppose $\alpha_{0} \in \Gamma_{t_{0}}\left[s \mid y_{0}\right]$. Then it is clear since $s \leq \tau$ that $\bar{\alpha} \in \Gamma_{t_{0}}\left[s \mid y_{0}\right]$. Hence

$$
\sup _{\alpha \in \Gamma_{\Gamma_{0}}\left(s y_{0}\right]} u(\alpha) \geq \sup _{\alpha \in \Gamma_{0_{0}}[s \mid y]} u_{\tau, Q_{s}}(\alpha)-\varepsilon
$$

and so $Q_{s}^{-}\left(t_{0}, x_{0}\right) \geq Q_{s, \tau}^{-}\left(t_{0}, x_{0} ; Q_{s}^{-}\right)$, i.e. (17) is established.

Corollary 3.4. If $\lim _{s \rightarrow 0} Q_{s}^{-}(t, x)=Q^{-}(t, x)$ uniformly on compacta then

$$
Q_{\tau}^{-}\left(t_{0}, x_{0} ; Q^{-}\right) \leq Q^{-}\left(t_{0}, x_{0}\right) \text {. }
$$

Similarly, if $\lim Q_{s}^{+}(t, x)=Q^{+}(t, x)$ uniformly on compacta then

$$
Q_{\tau}^{+}\left(t_{0}, x_{0} ; Q^{+}\right) \geq Q^{+}\left(t_{0}, x_{0}\right) \text {. }
$$

Proof. It is easy to show (see [2]) that every possible trajectory $(t, x(t))$ with $x\left(t_{0}\right)=x_{0}$ determined by (1) lies in a bounded subset $B$ of $R^{m+1}$. Define $\eta(s)=\sup _{(t, x) \in B}\left[Q^{-}(t, x)-Q_{s}^{-}(t, x)\right]$ so that by hypothesis $\lim _{s \rightarrow 0} \eta(s)=0$. Then clearly $Q_{s, r}^{-}\left(t_{0}, x_{0} ; Q^{-}\right)-Q_{s, r}^{-}\left(t_{0}, x_{0} ; Q_{s}^{-}\right) \leq \eta(s)$ and so

$$
\begin{aligned}
Q_{\tau}^{-}\left(t_{0}, x_{0} ; Q^{-}\right) & \leq \lim _{s \rightarrow 0}\left\{Q_{s, r}^{-}\left(t_{0}, x_{0} ; Q_{s}^{-}\right)+\eta(s)\right\} \\
& \leq \lim _{s \rightarrow 0}\left\{Q_{s}^{-}\left(t_{0}, x_{0}\right)+\eta(s)\right\}=Q^{-}\left(t_{0}, x_{0}\right) .
\end{aligned}
$$

This proves (18) and (19) follows by the dual argument.

\section{Lemma 3.5.}

$$
\begin{aligned}
& Q_{s, r}^{+}\left(t_{0}, x_{0} ; U\right) \leq Q_{s}^{+}\left(t_{0}, x_{0}\right), \\
& Q_{s, r}^{-}\left(t_{0}, x_{0} ; V\right) \geq Q_{s}^{-}\left(t_{0}, x_{0}\right)
\end{aligned}
$$

Proof. Again the proof of only one, (21), of the inequalities is supplied. Suppose $s>0$ is given. Since

$$
Q_{s}^{-}\left(t_{0}, x_{0}\right)=\inf _{y \in \mathcal{M}_{i}^{j}\left[t_{0}\right]} \sup _{\alpha \in I_{0}[s \mid y]} u(\alpha)
$$

given $\varepsilon>0$ there is an $\alpha \in \Gamma_{t_{0}}[s \mid y]$ such that

$$
u(\alpha) \geq Q_{s}^{-}\left(t_{0}, x_{0}\right)-\varepsilon / 3 \text {. }
$$

For $z \in \mathcal{M}_{2}\left(t_{0}\right)$,

$$
P(\alpha z, z) \geq Q_{s}^{-}\left(t_{0}, x_{0}\right)-\varepsilon / 3 .
$$

Now consider $u_{\tau, V}(\alpha)$; then there exists $z_{0} \in \mathcal{O} M_{2}\left(t_{0}\right)$ such that

$$
P_{r, V}\left(\alpha z_{0}, z_{0}\right) \leq u_{\tau, V}(\alpha)+\varepsilon / 3 \text {. }
$$


Write $\sigma z_{0}=\tau\left(\sigma z_{0}, z_{0}\right)$ and suppose $x_{0}(t)$ is the trajectory given by $\left(\alpha z_{0}, z_{0}\right)$. Then there is a strategy $\beta_{0} \in \Delta_{\sigma z_{0}}(0)$ in $G\left(\sigma z_{0}, x\left(\sigma z_{0}\right)\right)$ such that $v\left(\beta_{0}\right) \leq V\left(\sigma z_{0}\right.$, $\left.x\left(\sigma z_{0}\right)\right)+\varepsilon / 3$. Since $\alpha$ is a delay-strategy, $\alpha$ and $\beta_{0}$ can be played against each other from $\sigma z_{0}$ onwards (cf. [3, §3]). Therefore, there exists a $z \in \quad M_{2}\left(t_{0}\right)$ such that $\bar{z}(t)=z_{0}(t), t_{0} \leq t \leq \sigma z_{0}$, and $\left(\beta_{0} \alpha\right) \bar{z}(t)=\bar{z}(t), \sigma z_{0} \leq t$, where $\left(\beta_{0} \alpha\right) \bar{z}(t)$ is interpreted as $\beta_{0}$ acting on the restriction of $\alpha \bar{z}(t)$ to $\sigma z_{0} \leq t$. Then

$$
P(\alpha \bar{z}, \bar{z})=\int_{t_{0}}^{\sigma z_{0}} h\left(t, x_{0}(t), \alpha z_{0}(t), z_{0}(t)\right) d t+P^{*}(\alpha \bar{z}, \bar{z}),
$$

where $P *$ denotes the payoff in $G\left(\sigma z_{0}, x\left(\sigma z_{0}\right)\right)$. Thus

$$
\begin{aligned}
P(\alpha \bar{z}, \bar{z}) & \leq \int_{t_{0}}^{\sigma z_{0}} h d t+v\left(\beta_{0}\right) \\
& \leq \int_{t_{0}}^{\sigma z_{0}} h d t+V\left(\sigma z_{0}, x\left(\sigma z_{0}\right)\right)+\varepsilon / 3=P_{\tau, V}\left(\alpha z_{0}, z_{0}\right)+\varepsilon / 3 \\
& \leq u_{\tau, V}(\alpha)+2 \varepsilon / 3 \text { by }(23) .
\end{aligned}
$$

Substituting $\bar{z}$ into (22) we obtain $Q_{s}^{-}\left(t_{0}, x_{0}\right) \leq u_{\tau, V}(\alpha)+\varepsilon$, and therefore $Q_{s}^{-}\left(t_{0}, x_{0}\right) \leq Q_{s, r}^{-}\left(t_{0}, x_{0} ; V\right)$.

4. The Isaacs-Bellman equation. We first prove extensions to metric space valued functions of Lusin's theorem and Fillipov's lemma.

Lemma 4.1. Suppose $z:[0,1] \rightarrow Z$ is a measurable function with values in the compact metric space $Z$. Then for any $\varepsilon>0$ there is a subset $E \subset[0,1]$ with $m E>1-\varepsilon$ such that $z$ is continuous on $E$. ( $m$ denotes Lebesgue measure.)

Proof. As $Z$ is a compact metric space there is a countable dense subset $\left\{\xi_{k} ; k=1,2, \ldots\right\}$. Consider the functions $Q_{k}: Z \rightarrow R$ where $Q_{k}(z)=d\left(\xi_{k}, z\right)$, $k=1,2, \ldots, d$, denoting the metric on $Z$. By definition $z(t)$ measurable means $Q_{k}(z(t))$ is measurable in the usual Lebesgue sense, so by Lusin's theorem [6], for $\varepsilon / 2^{k}$ there is a subset $E_{k}$ of $[0,1]$ such that $Q_{k}(z(t))$ is continuous on $E_{k}$ and $m E_{k}>1-\varepsilon / 2^{k}, k=1,2, \ldots$ Put $E=\bigcap_{k=1}^{\infty} E_{k}$. Then $m E>1-\varepsilon$ and $Q_{k}(z(t))$ is continuous on $E$ for all $k=1,2, \ldots$ It is then immediate that $z(t)$ is continuous on $E$.

We shall need the following extension of Fillipov's lemma:

Lemma 4.2. $Y$ and $Z$ are compact metric spaces and $m: Y \times Z \rightarrow R$ is continuous. $z:[0, \tau] \rightarrow Z$ is measurable. Then there is a measurable function $\bar{y}:[0, \tau] \rightarrow Y$ such that $m(\bar{y}(t), z(t))=\max _{y \in Y} m(y, z(t))$ for almost all $t \in[0, \tau]$. Furthermore there is a strategy $\alpha \in \Gamma_{0}(0)$ such that $y=\alpha z$ for all $z \in M_{2}(0)$.

Proof. For any $\varepsilon>0$ by Lemma 4.1 there is a subset $E$ of $[0, \tau]$ such that $m E>\tau-\varepsilon$ and $z(t)$ is continuous on $E$. Write $M(z)=\max _{y \in Y} m(y, z)$ so that $M: Z \rightarrow R$ is continuous, and thus $M(z(t)) \in m(Y, z(t))$ is continuous on $E$.

As $Y$ is a compact metric space there is a continuous map $\kappa$ of the Cantor set $K$ onto $Y$ (see [7, Theorem 3.28]). We can, therefore, write $M(z(t))$ 
$=\max _{s \in K} m(\kappa(s), z(t))$. For each $t \in E$ denote by $K(t)$ the set of all $s \in K$ that satisfy $m(\kappa(s), z(t))=M(z(t))$. Continuity of $m$ and $\kappa$ implies that $K(t)$ is closed, and so compact. Write $u(t)=\min (s \in K(t))$. It remains to prove that $u(t)$ is measurable, and this will be the case if we can show that for any real $\lambda$ the set $\{t \in E ; u(t) \leq \lambda\}$ is closed. Suppose this is not the case; then there exists a sequence $t_{j} \in E$ such that $t_{j} \rightarrow \bar{t} \in E$ but $u\left(t_{j}\right) \leq \lambda<u(\bar{t})$. As $K$ is compact there is a subsequence $\left\{t_{j^{\prime}}\right\}$ of $\left\{t_{j}\right\}$ such that $u\left(t_{j^{\prime}}\right) \rightarrow \bar{u} \in K$ as $j^{\prime} \rightarrow \infty$, and we must have

$$
\underline{u}<u(\bar{t}) \text {. }
$$

Letting $t=t_{j}$, converge to $\bar{t}$ in the identity $m(\kappa(u(t)), z(t))=M(z(t))$ we have, by continuity on $E, m(\kappa(\bar{u}), z(\bar{t}))=M(z(\bar{t}))$. Thus the point $\bar{u}$ belongs to $K(\bar{t})$. But from the definition of $u(\bar{t}), u(\bar{t}) \leq \bar{u}$, which contradicts (24). Thus $\{t \in E ; u(t) \leq \lambda\}$ must be closed, and so $u(t)$ is measurable on $E$. Now take $\varepsilon=1 / n$ and denote the corresponding set $E$ by $E_{n}$. We see that $u(t)$ is measurable on each $E_{n}$ and so on their union $E^{*}$. As the measure of $E^{*}$ is $\tau$ we see $u(t)$ is measurable on $[0, \tau]$. Because the map $\kappa$ is continuous the composite function $\kappa \circ u(t)=\bar{y}(t)$ is measurable on $[0, \tau]$ and gives the required function $\bar{y}$. Note, by the method of selection, that $\bar{y}(t)$ depends only on $z(t)$ and so the map $\alpha$ sending $z$ to its corresponding $\bar{y}$ is a strategy in $\Gamma_{0}(0)$.

Suppose now the two players play a very simplified differential game $K(m ; \tau)$ where the payoff is of the form $P=\int_{0}^{\tau} m(y(t), z(t)) d t$. Here $\tau$ is fixed and $m: Y \times Z \rightarrow R$ is continuous. Thus in $K(m ; \tau)$ the dynamics are trivial and the form of the game does not alter with time. We determine various values of the game $K(m ; \tau)$.

Lemma 4.3. For $K(m ; \tau)$ we have

$$
Q^{+}=V^{+}=U=\tau\left\{\min _{z} \max _{y} m(y, z)\right\}
$$

and

$$
Q^{-}=V^{-}=V=\tau\left\{\max _{y} \min _{z} m(y, z)\right\}
$$

Proof. Suppose $J_{2}$ adopts a constant strategy: $\beta y(t) \equiv z_{0}(t) \equiv z_{0}$. Then

$$
P(y, \beta y)=\int_{0}^{\tau} m\left(y(t), z_{0}(t)\right) d t \leq \tau \max m\left(y, z_{0}\right)
$$

so

$$
V^{+}=Q^{+} \leq \tau \min _{z} \max _{y} m(y, z), \text { by Lemma 2.2. }
$$

Again write $M(z)=\max m(y, z)$ so $M: Z \rightarrow R$ is continuous and suppose 
$z(t) \in \mathcal{M}_{2}(0)$. By Lemma 4.2 there is a strategy $\alpha \in \Gamma_{0}(0)$ such that

$$
m(\alpha z(t), z(t))=M(z(t)) \text { for all } z \in \mathcal{M}_{2}(0) .
$$

Clearly $u(\alpha)=\tau \min _{z} M(z)=\tau \min _{z} \max _{y} m(y, z)$ and so

$$
U \geqslant \tau \min _{z} \max _{y} m(y, z) \text {. }
$$

From Lemma 2.1 and the remarks preceding it we see $Q^{+}=V^{+}=U=$ $\tau \min _{z} \max _{y} m(y, z)$. The dual result follows similarly.

We now return to the general game discussed in $\$ \S 2$ and 3. Our object is to show the relationship between the value functions $U, V, V^{-}, V^{+}, Q^{-}$and $Q^{+}$of $(t, x)$ and the solutions of certain partial differential equations associated with the system, first considered by Isaacs [8]. We define for the differential game associated with (1)-(4) the upper Isaacs-Bellman equation (in $R \times R^{m}-F$ ):

$$
L^{+} w \equiv \frac{\partial w}{\partial t}+\min _{z} \max _{y}(\nabla w \cdot f+h)=0
$$

and the lower Isaacs-Bellman equation

$$
L^{-} w \equiv \frac{\partial w}{\partial t}+\max _{y} \min _{z}(\nabla w \cdot f+h)=0,
$$

where both equations are subject to the boundary condition $w(t, x)=g(t, x)$ for $(t, x) \in \partial F, F$ being the terminal set.

Theorem 4.4. At points of differentiability we have

$$
L^{+}(U)=0, \quad L^{-}(V)=0, \quad L^{+}\left(V^{+}\right) \leq 0, \quad L^{-}\left(V^{-}\right) \geq 0 .
$$

Proof. Suppose $\left(t_{0}, x_{0}\right) \notin F$, the terminal set; then for some fixed $s_{0}>0$ we have, for any trajectory starting at $\left(t_{0}, x_{0}\right), t_{F} \geq t_{0}+s_{0}$. Furthermore, in the time $t_{0} \leq t \leq t_{0}+s_{0}$, all trajectories are contained in a bounded set $B$. Let

$$
\sup _{(, x) \in B} \operatorname{supsup}_{y \in Y}\|f(t, x, y, z)\|=M<\infty .
$$

Now suppose $\left(t_{0}, x_{0}\right)$ is a point of differentiability of $U$, so that

$$
U\left(t_{0}+s, x_{0}+\xi\right)=U\left(t_{0}, x_{0}\right)+s \partial U / \partial t+\xi \cdot \nabla U+(|s|+\|\xi\|) R(s, \xi) .
$$

Here $R$ is continuous at 0 and $R(0,0)=0$ and the partial derivatives are evaluated at $\left(t_{0}, x_{0}\right)$. Using (29) we deduce that, if $\left(t_{0}+s, x_{0}+\xi\right)$ is on a trajectory starting at $\left(t_{0}, x_{0}\right)$,

$$
\left|U\left(t_{0}+s, x_{0}+\xi\right)-U\left(t_{0}, x_{0}\right)-s \partial U / \partial t-\xi \cdot \nabla U\right| \leq s \eta(s),
$$

where $\lim _{s \rightarrow 0} \eta(s)=0$. Fixing $\tau=t_{0}+s$ where $s<s_{0}$, we have, by Theorem 3.1, $U\left(t_{0}, x_{0}\right)=U_{\tau}\left(t_{0}, x_{0} ; U\right)$. Write $W\left(t_{0}+s ; x\right)=U\left(t_{0}, x_{0}\right)+s \partial U / \partial t+\left(x-x_{0}\right)$ - $\nabla U$; then by (30) 


$$
\left|U_{\tau}\left(t_{0}, x_{0} ; U\right)-U_{\tau}\left(t_{0}, x_{0} ; W\right)\right| \leq s \eta(s) .
$$

Hence $\left|U\left(t_{0}, x_{0}\right)-U_{\tau}\left(t_{0}, x_{0} ; W\right)\right| \leq s \eta(s)$ and so $\left|U_{\tau}\left(t_{0}, x_{0} ; W_{0}\right)\right| \leq s \eta(s)$ where $W_{0}\left(t_{0}+s ; x\right)=s \partial U / \partial t+\left(x-x_{0}\right) \cdot \nabla U$. In the game $G_{r}\left(t_{0}, x_{0} ; W_{0}\right)$ the payoff corresponding to a pair of controls $y \in \mathcal{M}_{1}\left(t_{0}\right), z \in \mathcal{M}_{2}\left(t_{0}\right)$, is given by

$$
\begin{aligned}
P & =s \frac{\partial U}{\partial t}+\left(x-x_{0}\right) \cdot \nabla U+\int_{t_{0}}^{t_{0}+s} h(t, x, y(t), z(t)) d t \\
& =\int_{t_{0}}^{t_{0}+s}\left(\frac{\partial U}{\partial t}+f(t, x, y(t), z(t)) \cdot \nabla U+h(t, x, y(t), z(t))\right) d t
\end{aligned}
$$

where, of course, the partial derivatives are evaluated at $\left(t_{0}, x_{0}\right)$.

Now consider the game $K(m, s)$ where

$$
m(y, z)=\partial U / \partial t+f\left(t_{0}, x_{0}, y, z\right) \cdot \nabla U+h\left(t_{0}, x_{0}, y, z\right) .
$$

Clearly the payoff in $K(m, s)$ corresponding to the same pair of controls $P^{\prime}$ satisfies $\left|P^{\prime}-P\right| \leq s \delta(s)$ where $\lim _{s \rightarrow 0} \delta(s)=0$. Hence by Lemma 4.3

$$
\left|U_{\tau}\left(t_{0}, x_{0} ; W_{0}\right)-s L^{+} U\left(t_{0}, x_{0}\right)\right| \leq s \delta(s),
$$

and hence $\left|L^{+} U\left(t_{0}, x_{0}\right)\right| \leq \delta(s)+\eta(s)$, i.e. $L^{+} U\left(t_{0}, x_{0}\right)=0$.

The corresponding results for $V^{+}$and $V^{-}$follow from Theorem 3.2 in place of Theorem 3.1. The same problem for $Q^{+}$and $Q^{-}$depends on the weaker result Corollary 3.4. However, in this case we obtain

Theorem 4.5. Under the hypothesis of Corollary 3.4, at points of differentiability we have

$$
L^{+} Q^{+} \geq 0 \text { and } L^{-} Q^{-} \leq 0
$$

5. Comparison theorems. The results of this section are based on the following 'strategy selection theorems'. Suppose that $\varphi: R^{m+1} \times Y \times Z \rightarrow R$ is continuous and define

$$
\Phi(t, x)=\min _{z} \max _{y} \varphi(t, x, y, z) .
$$

Theorem 5.1. Let $G$ be the differential game given by (1)-(4). Then given a bounded subset $B$ of $(-\infty, T] \times R^{m}$ there exists a function $\eta:[0, \infty) \rightarrow[0, \infty)$ such that $\lim _{s \rightarrow 0} \eta(s)=0$ and with the following property: For every $\left(t_{0}, x_{0}\right) \in B$ there exists $\beta \in \Delta_{t_{0}}(s)$ such that whenever $\beta^{\prime} \in \Delta_{t_{0}}^{s}(\beta)$ and $y \in \mathcal{M} M_{1}\left(t_{0}\right)$, then for the trajectory $x^{\prime}(t)$ induced by $\left(y, \beta^{\prime} y\right)$ we have

$$
\varphi\left(t, x^{\prime}(t), y(t), \beta^{\prime} y(t)\right) \leq \Phi\left(t, x^{\prime}\right)+\eta(s)
$$

whenever $t_{0}+s \leq t \leq T$.

Proof. Every trajectory with initial point in $B$ is contained in a further bounded 
set $B^{\prime}$ for $t \leq T$ (see $\left.[2, \S 3]\right)$. Write $M=\sup _{B^{\prime} \times Y \times z}\|f(t, x, y, z)\|$. Suppose $x_{1}(t)$ and $x_{2}(t)$ is any pair of trajectories corresponding to the same pair of controls $(\gamma(t), z(t))$ but with initial conditions $\left(t_{0}, x_{1}\right)$ and $\left(t_{0}, x_{2}\right) \in B^{\prime}$. Then using (2) we obtain

$$
\begin{aligned}
\left\|x_{1}(t)-x_{2}(t)\right\| & \leq e^{K\left(t-t_{0}\right)}\left\|x_{1}-x_{2}\right\| \\
& \leq M^{\prime}\left\|x_{1}-x_{2}\right\|, \quad t_{0} \leq t \leq T,
\end{aligned}
$$

where $M^{\prime}=e^{K\left(T-t_{0}\right)}$. For $s>0$ let

$$
\sup \left|\varphi\left(t_{1}, x_{1}, y, z\right)-\varphi\left(t_{2}, x_{2}, y, z\right)\right|=\gamma(s),
$$

where the supremum is taken over $(y, z) \in Y \times Z$ and $\left(t_{1}, x_{1}\right)$ and $\left(t_{2}, x_{2}\right)$ in $B^{\prime}$ such that $\left|t_{2}-t_{1}\right| \leq 2 s,\left\|x_{2}-x_{1}\right\| \leq 2 M\left(M^{\prime}+1\right) s$. By uniform continuity of $\varphi$ on $B^{\prime} \times Y \times Z, \lim _{s \rightarrow 0} \gamma(s)=0$. Now fix $\left(t_{0}, x_{0}\right) \in B$ and let $t_{n}=t_{0}+n s$, $n=1,2, \ldots$ We define $\beta$ as follows: Let $z_{0}$ be any fixed control value in $Z$. Then for any $y \in O M_{1}\left(t_{0}\right)$ we let

$$
\beta y(t)=z_{0}, \quad t_{0} \leq t \leq t_{1} .
$$

Then we define

$$
\beta y(t)=z_{1}, \quad t_{1}<t \leq t_{2},
$$

where $z_{1}$ is chosen such that

$$
\max _{y} \varphi\left(t_{0}, x_{0}, y, z_{1}\right)=\Phi\left(t_{0}, x_{0}\right)
$$

The pair $(y(t), \beta y(t))$ induce a trajectory $x(t), t_{0} \leq t \leq t_{2}$, and we then choose $z_{2}$ such that

$$
\max _{y} \varphi\left(t_{1}, x\left(t_{1}\right), y, z_{2}\right)=\Phi\left(t_{1}, x\left(t_{1}\right)\right)
$$

and define $\beta y(t)=z_{2}, t_{2}<t \leq t_{3}$. We then repeat this process and eventually $\beta$ is defined so that

$$
\beta y(t)=z_{n}, \quad t_{n}<t \leq t_{n+1},
$$

where

$$
\max _{y} \varphi\left(t_{n-1}, x\left(t_{n-1}\right), y, z_{n}\right)=\Phi\left(t_{n-1}, x\left(t_{n-1}\right)\right) .
$$

Using Zorn's lemma $\beta$ is extended to the whole of $\mathcal{M}_{1}\left(t_{0}\right)$ as in Lemma 4.3. Clearly $\beta \in \Delta_{t_{0}}(s)$. Now suppose $\beta^{\prime} \in \Delta_{t_{0}}^{s}(\beta)$ so that $\beta^{\prime} y(t)=\beta y(t), t \geq t_{0}+s$. Let $x^{\prime}(t)$ be the trajectory induced by $\left(y, \beta^{\prime} y\right)$. Then

$$
\left\|x^{\prime}\left(t_{1}\right)-x\left(t_{1}\right)\right\| \leq 2 M s
$$


and so

$$
\left\|x^{\prime}(t)-x(t)\right\| \leq 2 M M^{\prime} s, \quad t_{0} \leq t \leq T .
$$

Now if $n \geq 1$ and $t_{n} \leq t \leq t_{n+1}$ then $\left\|x^{\prime}(t)-x\left(t_{n-1}\right)\right\| \leq 2 M M^{\prime} s+2 M s$ and $\left|t-t_{n-1}\right| \leq 2 s$, and so

$$
\left|\varphi\left(t, x^{\prime}(t), y(t), z_{n}\right)-\varphi\left(t_{n-1}, x\left(t_{n-1}\right), y(t), z_{n}\right)\right| \leq \gamma(s) .
$$

Clearly $\left|\Phi\left(t_{1} x^{\prime}(t)\right)-\Phi\left(t_{n-1}, x\left(t_{n-1}\right)\right)\right| \leq \gamma(s)$ and so

$$
\varphi\left(t, x^{\prime}(t), y(t), \beta^{\prime} y(t)\right) \leq \Phi\left(t, x^{\prime}(t)\right)+2 \lambda(s)
$$

for $t_{1} \leq t \leq T$.

Theorem 5.2. For every $\left(t_{0}, x_{0}\right)$ with $t_{0} \leq T$ and every $\varepsilon>0$ there exists a strategy $\alpha \in \Gamma_{t_{0}}$ such that if $z \in M_{2}\left(t_{0}\right)$ and $x(t)$ is the trajectory corresponding to $(\alpha z, z)$

$$
\varphi(t, x(t), \alpha z(t), z(t)) \geq \Phi(t, x(t))-\varepsilon \quad \text { a.e. }
$$

for $t_{0} \leq t \leq T$.

Proof In Lemma 4.3 we showed that if $m: Y \times Z \rightarrow R$ is continuous there exists a strategy $\alpha$ for $J_{1}, \alpha: M_{2}(0) \rightarrow \propto M_{1}(0)$ such that

$$
m(\alpha z(t), z(t)) \geq \min _{z} \max _{y} m(y, z) \text { a.e. } t \geq 0 ;
$$

for any $z \in M_{2}(0)$. Thus, by the same reasoning, for any $(\bar{t}, \bar{x}) \in R^{m+1}$ there is a strategy $\alpha[\bar{t}, \bar{x}]: \propto M_{2}\left(t_{0}\right) \rightarrow \propto M_{1}\left(t_{0}\right)$ such that

$$
\varphi(\bar{t}, \bar{x}, \alpha[\bar{t}, \bar{x}] z(t), z(t)) \geq \Phi(\bar{t}, \bar{z}) \text { a.e. } t \geq \bar{t} .
$$

For $s>0$ and $\left(t_{0}, x_{0}\right)$ fixed we define $t_{n}=t_{0}+n s$ and construct $\alpha_{s} \in \Gamma_{t_{0}}$; thus

$$
\alpha_{s} z(t)=\alpha\left[t_{0}, x_{0}\right] z(t), \quad t_{0} \leq t \leq t_{1} .
$$

Then $\left(\alpha_{s} z, z\right)$ determines a trajectory $x(t), t_{0} \leq t \leq t_{1}$. We define $\alpha_{s} z(t)=\alpha\left[t_{1}\right.$, $\left.x\left(t_{1}\right)\right] z(t), t_{1}<t \leq t_{2}$, and proceed inductively. Clearly $\alpha_{s} \in \Gamma_{t_{0}}$ and

$$
\varphi\left(t_{n}, x\left(t_{n}\right), \alpha_{s} z(t), z(t)\right) \geq \Phi\left(t_{n}, x\left(t_{n}\right)\right) \text { a.e. }
$$

for $t_{n} \leq t \leq t_{n+1}$ and $n \geq 0$. Using the uniform continuity of $\varphi$ as in Theorem 5.1 we obtain for $t_{0} \leq t$

$$
\varphi\left(t, x(t), \alpha_{s} z(t), z(t)\right) \geq \Phi(t, x(t))-\eta(s) \text { a.e. }
$$

where $\lim _{s \rightarrow 0} \eta(s)=0$. This proves (33).

We now come to the main theorem of the section, in which we compare a $C^{1}$ function $\theta$ satisfying either $L^{+} \theta \geq 0$ or $L^{+} \theta \leq 0$ everywhere with the value of the 
game $G$. Results of this type were first considered by Rao [11] and later by Friedman [5]. However, in [11] Rao restricts his attention to fixed time games and Friedman, who approximates by solutions of parabolic equations, requires that $\theta$ is $C^{1}$ and piecewise $C^{2}$, together with his condition $(F)$. The referee tells us that Rao considered comparison theorems for pursuit-evasion games in his thesis (University of Rhode Island, 1971).

Theorem 5.3. Let $G$ be the game given by (1)-(4) and suppose $\theta$ is a continuous function on $R^{m+1}$ which is $C^{1}$ on $R^{m+1}-$ int $F$. Suppose

(a) $\theta(t, x) \geq g(t, x),(t, x) \in \partial F$,

(b) $L^{+} \theta \leq 0,(t, x) \in R^{m+1}-$ int $F$.

Then

$$
\theta(t, x) \geq Q^{+}(t, x), \quad(t, x) \in R^{m+1}-\text { int } F,
$$

and for any bounded subset $B$ of $R^{m+1}-$ int $F$ there exists a function $\eta:[0, \infty)$ $\rightarrow[0, \infty)$ such that $\lim _{s \rightarrow 0} \eta(s)=0$ and

$$
\theta(t, x) \geq Q_{s}^{+}(t, x)-\eta(s), \quad(t, x) \in R^{m+1}-\text { int } F .
$$

Proof. Let $\varphi(t, x, y, z)=\partial \theta / \partial t+f \cdot \nabla \theta+h$ for $(t, x, y, z) \in\left(R^{m+1}-\right.$ int $\left.F\right) \times Y$ $\times Z$ and extend $\varphi$ to be defined and continuous on $R^{m+1} \times Y \times Z$. Then

$$
\begin{aligned}
\Phi(t, x) & =\min _{z} \max _{y} \varphi(t, x, y, z) \\
& =L^{+} \theta, \quad(t, x) \in R^{m+1}-\text { int } F .
\end{aligned}
$$

We now quote Theorem 5.1: Suppose $B$ is a bounded subset of $R^{m+1}-$ int $F$ and $\eta$ is the function determined by Theorem 5.1. For $s>0$ and $\left(t_{0}, x_{0}\right) \in B$ choose $\beta \in \Delta_{t_{0}}(s)$ as in Theorem 5.1 and let $\beta^{\prime} \in \Delta_{t_{0}}^{s}(\beta)$. Then if $y \in M_{1}\left(t_{0}\right)$ and $x^{\prime}(t)$ is the trajectory corresponding to $\left(y, \beta^{\prime} y\right)$,

$$
\varphi\left(t, x^{\prime}(t), y(t), \beta^{\prime} y(t)\right) \leq \Phi\left(t, x^{\prime}(t)\right)+\eta(s)
$$

for $t_{0}+s \leq t \leq T$ (equation (32)).

For $t_{0}+s \leq t \leq t_{F}$ (if $\left.t_{F}>t_{0}+s\right)$ we have $\varphi\left(t, x^{\prime}(t), y(t), \beta^{\prime} y(t)\right) \leq \eta(s)$.

Suppose, as before, that every trajectory with initial point in $B$ is contained in the bounded set $B^{\prime}$ and that

$$
\sup _{(, x) \in B^{\prime}} \sup _{(y, z) \in Y \times Z}|\varphi(t, x, y, z)|=M^{\prime \prime},
$$

as $\varphi$ is continuous. Then

$$
\int_{t_{0}}^{t_{F}} \varphi\left(t, x^{\prime}(t), y(t), \beta^{\prime} y(t)\right) d t \leq M^{\prime \prime} s+\left(T-t_{0}\right) \eta(s) .
$$

However 


$$
\begin{aligned}
& \int_{t_{0}}^{t_{F}} \varphi\left(t, x^{\prime}(t), y(t), \beta^{\prime} y(t)\right) d t \\
& =\int_{t_{0}}^{t_{F}}\left(\frac{\partial \theta}{\partial t}+f \cdot \nabla \theta+h\right) d t \\
& =\int_{t_{0}}^{t_{F}} \frac{d}{d t} \theta\left(t, x^{\prime}(t)\right) d t+\int_{t_{0}}^{t_{F}} h d t \\
& =\theta\left(t_{F}, x^{\prime}\left(t_{F}\right)\right)-\theta\left(t_{0}, x_{0}\right)+\int_{t_{0}}^{t_{F}} h d t .
\end{aligned}
$$

We use here Lemma 3.22 of Friedman [4], that $t \mapsto \theta(t, x(t))$ is absolutely continuous and

$$
\frac{d \theta(t, x(t))}{d t}=\frac{\partial \theta}{\partial t}+\dot{x} \cdot \nabla \theta
$$

Thus by (37) and (38)

$$
\begin{aligned}
P\left(y, \beta^{\prime} y\right) & =g\left(t_{F}, x^{\prime}\left(t_{F}\right)\right)+\int_{t_{0}}^{t_{F}} h d t \\
& \leq \theta\left(t_{F}, x^{\prime}\left(t_{F}\right)\right)+\int_{t_{0}}^{t_{F}} h d t \\
& =\theta\left(t_{0}, x_{0}\right)+\int_{t_{0}}^{t_{F}} \varphi\left(t, x^{\prime}(t), y(t), \beta^{\prime} y(t)\right) d t \\
& \leq \theta\left(t_{0}, x_{0}\right)+M^{\prime \prime} s+\left(T-t_{0}\right) \eta(s) .
\end{aligned}
$$

Hence

$$
\begin{aligned}
& v\left(\beta^{\prime}\right) \leq \theta\left(t_{0}, x_{0}\right)+M^{\prime \prime} s+\left(T-t_{0}\right) \eta(s), \\
& v_{s}(\beta) \leq \theta\left(t_{0}, x_{0}\right)+M^{\prime \prime} s+\left(T-t_{0}\right) \eta(s),
\end{aligned}
$$

and so by the remarks preceding Lemma 2.1

$$
Q_{s}^{+}\left(t_{0}, x_{0}\right) \leq \theta\left(t_{0}, x_{0}\right)+\eta^{\prime}(s)
$$

where $\eta^{\prime}(s)=M s+\left(T-t_{0}\right) \eta(s)$. This proves (35) and (34) follows immediately.

We remark that as in Friedman [5], we may improve Theorem 5.3 by observing that since every trajectory emanating from a fixed point $\left(t_{0}, x_{0}\right)$ is contained in a bounded set $B^{\prime \prime}$ we need only insist that $L^{+} \theta \leq 0$ on $B^{\prime \prime}$ to obtain $\theta\left(t_{0}, x_{0}\right)$ $\geq Q^{+}\left(t_{0}, x_{0}\right)$.

We now prove the converse theorem using Theorem 5.2:

Theorem 5.4. Suppose $G$ is given by (1)-(4) and $\theta$ is continuous on $R^{m+1}$ and continuously differentiable on $R^{m+1}-$ int $F$. Suppose 
(a)

(b)

Then

$$
\begin{aligned}
\theta(t, x) & \leq g(t, x), & & (t, x) \in \partial F, \\
L^{+} \theta & \geq 0, & & (t, x) \in R^{m+1}-\text { int } F .
\end{aligned}
$$

$\theta(t, x) \leq g(t, x), \quad(t, x) \in \partial F$,

$L^{+} \theta \geq 0, \quad(t, x) \in R^{m+1}-$ int $F$.

Proof. We omit the proof as it is almost the same as that of Theorem 5.3, using Theorem 5.2 instead of Theorem 5.1.

Naturally, both the above theorems may be stated for $L^{-}$with $Q^{-}$replacing $Q^{+}$ and $V$ replacing $U$.

Theorem 5.5. Suppose there is a $C^{1}$-solution $\theta$ of the upper Isaacs-Bellman equation (27) (i.e. $\left.L^{+} \theta=0\right)$, with $\theta=g$ on $\partial F$. Then $\theta(t, x) \equiv U(t, x) \equiv V^{+}(t, x)$ $=Q^{+}(t, x)$.

Proof. By 5.3 we obtain $\theta \geq Q^{+}$and by 5.4 we obtain $\theta \leq U$. As $U \leq Q^{+}$we obtain the result.

6. Continuity of $V^{+}$and $Q^{+}$. Thus far we have not referred to the problem of continuity of the functions $V^{+}$and $Q^{+}$. In general (see \$2) we may expect that $V^{+}$and $Q^{+}$are discontinuous. The behavior of the functions near the boundary turns out to be critical. Although we omit the proof, which uses Theorem 3.1, we may observe that $U$ is continuous if and only if $U$ is continuous at each point of $\partial F$ (i.e. iff $U(t, x) \rightarrow U\left(t^{\prime}, x^{\prime}\right)=g\left(t^{\prime}, x^{\prime}\right)$ whenever $\left.(t, x) \rightarrow\left(t^{\prime}, x^{\prime}\right) \in \partial F\right)$.

Define for each $(t, x) \in R^{m+1}-$ int $F$

$$
\bar{Q}_{s}^{+}(t, x)=\limsup _{(r, \xi) \rightarrow(t, x)} Q_{s}^{+}(t, x) .
$$

Then we have immediately that for each $s>0$ the function $\bar{Q}_{s}^{+}$is upper semicontinuous.

For $R>0$ write $B_{R}=\{(x, t):\|x\|+|t| \leq R\}$.

Lemma 6.1. Suppose for $(t, x) \in B_{R} \cap \partial F$

$$
\lim _{s \rightarrow 0} \bar{Q}_{s}^{+}(t, x)=g(t, x) .
$$

Then there exists a monotonic increasing function $\eta:[0, \infty) \rightarrow[0, \infty)$ with $\lim _{\gamma \rightarrow 0} \eta(\gamma)$ $=0$, such that

$$
Q_{s}^{+}(t, x) \leq g(t, x)+\eta(s+\rho)
$$

for $(t, x) \in B_{R}$. Here $\rho=\rho(t, x)$ is the distance of $(t, x)$ from $F$.

Proof. (For $(t, x) \in B_{R} \cap \partial F,(42)$ is simply Dini's theorem since each $\bar{Q}_{s}^{+}$is upper semicontinuous.) Define 


$$
\eta(\gamma)=\sup _{s+\rho \leq \gamma}\left(Q_{s}^{+}(t, x)-g(t, x)\right) .
$$

We must show that $\lim _{\gamma \rightarrow 0} \eta(\gamma)=0$. If not, there exist sequences $s_{n} \rightarrow 0$ and $\left(t_{n}, x_{n}\right) \in B_{R}$ such that

$$
\inf \left(Q_{s_{n}}^{+}\left(t_{n}, x_{n}\right)-g\left(t_{n}, x_{n}\right)\right)=\varepsilon>0,
$$

and $\lim _{n \rightarrow \infty} \rho\left(t_{n}, x_{n}\right)=0$. By selecting a subsequence we may suppose that $\left(t_{n}, x_{n}\right) \rightarrow\left(t_{0}, x_{0}\right)$. Clearly $\rho\left(t_{0}, x_{0}\right)=0$, i.e. $\left(t_{0}, x_{0}\right) \in F$. For $\sigma>0$ there exists $n_{0}$ such that for $n \geq n_{0}, s_{n} \leq \sigma$. Then $Q_{s_{n}}^{+}\left(t_{n}, x_{n}\right) \leq Q_{\sigma}^{+}\left(t_{n}, x_{n}\right), n \geq n_{0}$, and so $\bar{Q}_{\sigma}\left(t_{0}, x_{0}\right) \geq g\left(t_{0}, x_{0}\right)+\varepsilon$ for all $\sigma>0$, contrary to assumption (41).

Definition 6.2. A point $(t, x)$ of $\partial F$ is $Q^{+}$-regular if it satisfies (41) and $U$ is continuous at $(t, x)$, i.e. $\lim _{(\tau, \xi) \rightarrow(t, x)} U(\tau, \xi)=U(t, x)=g(t, x)$. We shall say $\partial F$ is $Q^{+}$regular if every point of $\partial F$ is $Q^{+}$regular.

In order to prove the next theorem it will be necessary to replace the Lipschitz condition (2) in $x$ by a Lipschitz condition in both $t$ and $x$ :

$$
\left\|f\left(t_{1}, x_{1}, y, z\right)-f\left(t_{2}, x_{2}, y, z\right)\right\| \leq K\left(\left|t_{1}-t_{2}\right|+\left\|x_{1}-x_{2}\right\|\right) .
$$

Theorem 6.3. Under the Lipschitz condition (43), suppose $\partial F$ is $Q^{+}-$regular, then $Q^{+}$is continuous (on $R^{m+1}-$ int $F$ ) and

$$
\lim _{s \rightarrow 0} Q_{s}^{+}(t, x)=Q^{+}(t, x)
$$

uniformly on compacta.

Proof. Note if $(t, x) \in \partial F$ the continuity of $Q^{+}(t, x)$ follows from Lemma 6.1 and Definition 6.2. Consider the set $C=B_{r} \cap\left(R^{m+1}-\right.$ int $\left.F\right)$ for some $r>0$. Then any trajectory with initial point in $C$ is contained, for $t \leq T$, in a larger compact set $B_{R}$.

First we observe that $g$ and $h$ are uniformly continuous on $B_{R}$ and $B_{R} \times Y \times Z$ respectively. Thus there exists a function $A_{1}(\delta)$ with $\lim _{s \rightarrow 0} A_{1}(\delta)=0$ and

$$
\begin{aligned}
\left|g\left(t_{1}, x_{1}\right)-g\left(t_{2}, x_{2}\right)\right| & \leq A_{1}(\delta), \\
\left|h\left(t_{1}, x_{1}, y, z\right)-h\left(t_{2}, x_{2}, y, z\right)\right| & \leq A_{1}(\delta),
\end{aligned}
$$

whenever

$$
\left|t_{1}-t_{2}\right|+\left\|x_{1}-x_{2}\right\| \leq \delta e^{K(T+R)}
$$

Next we set

$$
A_{2}(\delta)=\eta\left(\delta e^{K(T+R)}\right)
$$

where $\eta$ is defined as in Lemma 6.1. Finally on $B_{R}$ we can determine $A_{3}(\delta)$ such that $\lim _{\delta \rightarrow 0} A_{3}(\delta)=0$ and 


$$
g(t, x)-U(t, x) \leq A_{3}(\delta)
$$

whenever $\rho(t, x) \leq \delta e^{K(T+R)}$. This follows by a simple compactness argument.

Now suppose $\left(t_{1}, x_{1}\right)$ and $\left(t_{2}, x_{2}\right) \in B_{R}-F$ and write

$$
\sigma=t_{2}-t_{1}, \quad \delta=\left|t_{2}-t_{1}\right|+\left\|x_{2}-x_{1}\right\| .
$$

Then if $y=y(t) \in \mathcal{\prime} M_{1}\left(t_{1}\right)$ and $z=z(t) \in \mathcal{\prime} M_{2}\left(t_{1}\right)$ we can determine naturally

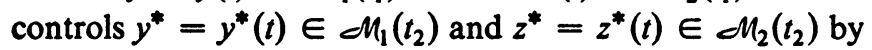

$$
y^{*}(t)=y(t-\sigma), \quad z^{*}(t)=z(t-\sigma) .
$$

The mapping $(y, z) \mapsto\left(y^{*}, z^{*}\right)$ is a nonanticipating invertible map $\mathcal{M}_{1}\left(t_{1}\right)$ $\times M_{2}\left(t_{1}\right) \rightarrow M_{1}\left(t_{2}\right) \times \mathcal{M}_{2}\left(t_{2}\right)$ and induces a map between the sets of strategies for each player in $G\left(t_{1}, x_{1}\right)$ and $G\left(t_{2}, x_{2}\right)$. Suppose $\xi_{1}(t)$ and $\xi_{2}(t)$ are trajectories corresponding to $(y, z)$ and $\left(y^{*}, z^{*}\right)$ respectively, with initial conditions $\xi_{1}\left(t_{1}\right)$ $=x_{1}, \xi_{2}\left(t_{2}\right)=x_{2}$. Then

(49) $\frac{d}{d t}\left(\xi_{1}(t)-\xi_{2}(t+\sigma)\right)=f\left(t, \xi_{1}(t), y(t), z(t)\right)-f\left(t+\sigma, \xi_{2}(t+\sigma), y(t), z(t)\right)$

and we may show that

$$
\left\|\xi_{1}(t)-\xi_{2}(t+\sigma)\right\| \leq \delta e^{K\left(t-t_{1}\right)}-|\sigma| \text { for } t_{1} \leq t \leq T .
$$

Hence

$$
\left\|\xi_{1}(t)-\xi_{2}(t+\sigma)\right\| \leq \delta e^{K(T+R)}-|\sigma| \text { for } t_{1} \leq t \leq T .
$$

Next we define a stopping time $\tau$ : $\mathcal{M}_{1}\left(t_{1}\right) \times M_{1}\left(t_{2}\right) \rightarrow R$ by $\tau=\tau(y, z)$ where

$$
\min \left\{\rho\left(\tau, \xi_{1}(\tau)\right) ; \rho\left(\tau+\sigma, \xi_{2}(\tau+\sigma)\right)\right\}=0
$$

but

$$
\min \left\{\rho\left(t, \xi_{1}(t)\right) ; \rho\left(t+\sigma, \xi_{2}(t+\sigma)\right)\right\} \neq 0
$$

for $t_{1} \leq t<\tau$. Then $\tau$ is a nonanticipating stopping time, and $\tau \leq T$. Hence by (50)

$$
\max \left\{\rho\left(\tau, \xi_{1}(\tau)\right) ; \rho\left(\tau+\sigma, \xi_{2}(\tau+\sigma)\right)\right\} \leq \delta e^{K(T+R)} .
$$

Write $\sup _{(t, x) \in B_{R}} \sup _{y \in Y} \sup _{z \in Z}\|f(t, x, y, z)\|=M_{R}$ and $t_{R}=\min \left(\rho\left(t_{1}, x_{1}\right) M_{R}^{-1}\right.$, $\left.\rho\left(t_{2}, x_{2}\right) M_{R}^{-1}\right)$. Suppose $s<t_{R} \leq \tau$. Now by Theorem 3.3, equation (16),

$$
\begin{aligned}
Q_{s}^{+}\left(t_{1}, x_{1}\right) & \leq Q_{s, \tau}^{+}\left(t_{1}, x_{1} ; Q_{s}^{+}\right) \\
& \leq Q_{s, \tau}^{+}\left(t_{1}, x_{1} ; g\right)+\eta\left(\delta e^{K(T+R)}+s\right) \quad \text { by Lemma } 6.1 \\
& \leq Q_{s, \tau}^{+}\left(t_{1}, x_{1} ; g\right)+A_{2}(\delta+s) \quad \text { by }(47) .
\end{aligned}
$$


Now $\left|g\left(\tau+\sigma, \xi_{2}(\tau+\sigma)\right)-g\left(\tau, \xi_{1}(\tau)\right)\right| \leq A_{1}(\delta)$ and

$$
\begin{gathered}
\left|\int_{t_{1}}^{\tau} h\left(t_{1}, \xi_{1}(t), y(t), z(t)\right) d t-\int_{t_{2}}^{\tau+\sigma} h\left(t, \xi_{2}(t), y^{*}(t), z^{*}(t)\right) d t\right| \\
\leq \int_{t_{1}}^{\tau} A_{1}(\delta) d t \leq(r+T) A_{1}(\delta) .
\end{gathered}
$$

Thus

$$
Q_{s, r}^{+}\left(t_{1}, x_{1} ; g\right) \leq Q_{s, \tau+\sigma}^{+}\left(t_{2}, x_{2} ; g\right)+(1+r+T) A_{1}(\delta) .
$$

So by (48)

$$
Q_{s, \tau+\sigma}^{+}\left(t_{2}, x_{2} ; g\right) \leq Q_{s, \tau+\sigma}^{+}\left(t_{2}, x_{2} ; U\right)+A_{3}(\delta) .
$$

Finally, by Lemma 3.5 ,

$$
Q_{s, r+\sigma}^{+}\left(t_{2}, x_{2} ; U\right) \leq Q_{s}^{+}\left(t_{2}, x_{2}\right) .
$$

Combining (51), (52), (53) and (54):

$$
Q_{s}^{+}\left(t_{1}, x_{1}\right) \leq Q_{s}^{+}\left(t_{2}, x_{2}\right)+(1+r+T) A_{1}(\delta)+A_{2}(\delta+s)+A_{3}(\delta) .
$$

By symmetry

(56) $\left|Q_{s}^{+}\left(t_{1}, x_{1}\right)-Q_{s}^{+}\left(t_{2}, x_{2}\right)\right| \leq(1+r+T) A_{1}(\delta)+A_{3}(\delta)+A_{2}(\delta+s)$.

Letting $s \rightarrow 0$, if $A_{4}(\delta)=(1+r+T) A_{1}(\delta)+A_{3}(\delta)$

$$
\left|Q^{+}\left(t_{1}, x_{1}\right)-Q^{+}\left(t_{2}, x_{2}\right)\right| \leq A_{4}(\delta)+A_{2}(2 \delta)
$$

so that $Q^{+}$is continuous.

Let $M_{s}(\varepsilon)=\left\{(t, x) \in C: Q_{s}^{+}(t, x)-Q^{+}(t, x) \geq \varepsilon\right\}$. Then as $Q_{s}^{+}$is monotone decreasing the sets $M_{s}(\varepsilon)$ are monotone decreasing in $s$ and $\cap_{s>0} M_{s}(\varepsilon)=\varnothing$. Suppose $(t, x) \in \overline{M_{s}(\varepsilon)} \cap\left(B_{R}-F\right)$ so that for some $\left(t_{0}, x_{0}\right) \in M_{s}(\varepsilon) \cap\left(B_{R}\right.$ $-F)$

$$
\left|t-t_{0}\right|+\left\|x-x_{0}\right\| \leq \delta
$$

Then

$$
\begin{aligned}
Q_{s}^{+}(t, x)-Q^{+}(t, x) \geq & Q_{s}^{+}\left(t_{0}, x_{0}\right)-Q^{+}\left(t_{0}, x_{0}\right) \\
& -2 A_{4}(\delta)-A_{2}(2 \delta)-A_{2}(\delta+s) \\
\geq & \varepsilon-2 A_{4}(\delta)-A_{2}(2 \delta)-A_{2}(\delta+s) .
\end{aligned}
$$

As $\delta$ is arbitrary $Q_{s}^{+}(t, x)-Q^{+}(t, x) \geq \varepsilon-A_{2}(2 s)$. By Lemma 6.1 and Definition 6.2 a similar inequality holds when $(t, x) \in \overline{M_{s}(\varepsilon)} \cap \partial F$. Because $\lim _{s \rightarrow 0} A_{2}(2 s)$ $=0$ we conclude that $\cap_{s>0} \overline{M_{s}(\varepsilon)}=\varnothing$. By the compactness of $C$ there exists $s_{0}$ 
such that $M_{s_{0}}(\varepsilon)=\varnothing$, i.e. $Q_{s}^{+}(t, x)-Q^{+}(t, x)<\varepsilon$ for $s \leq s_{0}$. Hence $\lim _{s \rightarrow 0} Q_{s}^{+}$ $=Q^{+}$uniformly on $C$.

Theorem 6.4. If the Lipschitz condition (43) is satisfied and $\partial F$ is $Q^{+}$-regular then $Q^{+}=V^{+}$.

Proof. Let $\left(t_{0}, x_{0}\right) \in R^{m+1}-F$ and suppose $Q^{+}\left(t_{0}, x_{0}\right)-V^{+}\left(t_{0}, x_{0}\right)=\varepsilon>0$. Then every trajectory in $G$ with initial point at $\left(t_{0}, x_{0}\right)$ is contained in $B_{R}$ for some $R>0$.

Write $M=\sup _{B_{R} \times Y \times Z}\|f(t, x, y, z)\|$. We determine inductively a sequence $\left(t_{n}, x_{n}\right)$ in $B_{R}$ for $n \geq 1$ with $\rho\left(t_{n}, x_{n}\right)>0$ for all $n$. Suppose $\left(t_{k}, x_{k}\right)$ has been determined. Then let

$$
\tau_{k}=t_{k}+(2(M+1))^{-1} \rho\left(t_{k}, x_{k}\right),
$$

and consider the game $G_{\tau_{k}}\left(t_{k}, x_{k} ; Q^{+}\right)$. Then by Theorem 6.3 and Corollary 3.4

$$
Q^{+}\left(t_{k}, x_{k}\right) \leq Q_{\tau_{k}}^{+}\left(t_{k}, x_{k} ; Q^{+}\right) .
$$

However, $G_{\eta_{k}}\left(t_{k}, x_{k} ; Q^{+}\right)$is a game of fixed duration, and $Q^{+}$is continuous, so that, by Lemma $2.2, Q^{+}\left(t_{k}, x_{k}\right) \leq V_{\tau_{k}}^{+}\left(t_{k}, x_{k} ; Q^{+}\right)$. However, by Theorem 3.2, $V^{+}\left(t_{k}, x_{k}\right) \geq V_{\tau_{k}}^{+}\left(t_{k}, x_{k} ; V^{+}\right)$. It follows that there exists a trajectory $\xi(t)$ in $G_{\tau_{k}}\left(t_{k}, x_{k}\right) \quad$ with $Q^{+}\left(t_{k+1}, x_{k+1}\right)-V^{+}\left(t_{k+1}, x_{k+1}\right) \geq Q^{+}\left(t_{k}, x_{k}\right)-V^{+}\left(t_{k}, x_{k}\right)$ $-\varepsilon / 2^{k+2}$, where $t_{k+1}=\tau_{k}$ and $\xi\left(t_{k+1}\right)=x_{k+1}$. Furthermore $\rho\left(t_{k+1}, x_{k+1}\right) \geq \rho\left(t_{k}\right.$, $\left.x_{k}\right)-(M+1)\left(t_{k+1}-t_{k}\right)>0$. Also $\left(t_{n}, x_{n}\right)$ is a sequence in $B_{R}$ as each $\left(t_{n}, x_{n}\right)$ can be reached from $\left(t_{0}, x_{0}\right)$ by a trajectory. By construction

$$
Q^{+}\left(t_{n}, x_{n}\right)-V^{+}\left(t_{n}, x_{n}\right) \geq \varepsilon\left(1-\sum_{k=1}^{n} 2^{-(k+1)}\right) \geq \varepsilon / 2 .
$$

For each $n,\left(t_{n}, x_{n}\right) \notin F$ so that $t_{n} \leq T$ for all $n$. Hence

$$
\lim _{n \rightarrow \infty} t_{n}=t_{0}+\sum_{k-1}^{\infty}\left(t_{k+1}-t_{k}\right)=\bar{t}
$$

exists and we have $\lim _{k \rightarrow \infty}\left(t_{k+1}-t_{k}\right)=0$. By (57), $\lim _{k \rightarrow \infty} \rho\left(t_{k}, x_{k}\right)=0$.

Clearly since $\left\|x_{k+1}-x_{k}\right\| \leq M\left(t_{k+1}-t_{k}\right)$ the sequence $x_{k}$ converges to $\bar{x}$ say. Then $\rho(\bar{t}, \bar{x})=0$, i.e. $(\bar{t}, \bar{x}) \in \cdot F$. Hence

$$
\begin{aligned}
\lim _{k \rightarrow \infty}( & \left.Q^{+}\left(t_{k}, x_{k}\right)-V^{+}\left(t_{k}, x_{k}\right)\right) \\
& \leq \lim _{k \rightarrow \infty}\left(Q^{+}\left(t_{k}, x_{k}\right)-U\left(t_{k}, x_{k}\right)\right) \\
& =0 \text { by Theorem } 6.3 \text { and the regularity of } \partial F .
\end{aligned}
$$

However, this contradicts (58), and so $Q^{+}\left(t_{0}, x_{0}\right)=V^{+}\left(t_{0}, x_{0}\right)$.

7. Criteria for regularity. In this section we present two convenient tests for the $Q^{+}$-regularity of the boundary $\partial F$. The first is based on the comparison theorems 
of $\$ 5$ whilst the second is based on a similar criterion for the regularity of the boundary for the Laplacian operator $\nabla^{2}$ given by Perron [10] (see also Krzyzański $[9$, pp. 377-396]).

Theorem 7.1. Suppose that there exist functions $\theta_{1}, \theta_{2}$ both $C^{1}$ on $R^{m+1}-$ int $F$, such that $\theta_{1}=\theta_{2}=g$ on $\partial F$ and $L^{+} \theta_{1} \leq 0 \leq L^{+} \theta_{2}$ on $R^{m+1}-$ int $F$. Then $\partial F$ is $Q^{+}$-regular.

Proof. By Theorem 5.3, equation (35), on any bounded set $B \subset R^{m+1}-$ int $F$

$$
\bar{Q}_{s}^{+} \leq \theta_{1}(t, x)+\eta(s), \quad(t, x) \in \partial F .
$$

Hence $\bar{Q}^{+}(t, x)=g(t, x),(t, x) \in \partial F$, where $\bar{Q}^{+}=\lim _{s \rightarrow 0} \bar{Q}_{s}^{+}$. Similarly, by Theorem 5.4,

$$
U(t, x) \geq \theta_{2}, \quad(t, x) \in R^{m+1}-\text { int } F
$$

and so

$$
\liminf _{(\tau, \xi) \rightarrow(t, x)} U(\tau, \xi) \geq g(t, x), \quad(t, x) \in \partial F
$$

But

$$
\lim _{(\tau, \xi) \rightarrow(t, x)} \sup _{(\tau, \xi)} \leq \bar{Q}^{+}(t, x)=g(t, x) .
$$

Therefore, $U$ is continuous at each point of $\partial F$.

The next criterion involves not the operator $L^{+}$but the operator $L_{0}^{+}$involving only the dynamics of the game:

$$
L_{0}^{+} \psi=\frac{\partial \psi}{\partial t}+\min _{z} \max _{y}(\nabla \psi \cdot f) .
$$

Theorem 7.2. Let $\left(t_{0}, x_{0}\right) \in \partial F$ and suppose there is a neighborhood $N$ of $\left(t_{0}, x_{0}\right)$ and there exist $C^{1}$ functions $\theta_{1}, \theta_{2}$ on $N$ such that

(i) $\theta_{1}\left(t_{0}, x_{0}\right)=\theta_{2}\left(t_{0}, x_{0}\right)=0, \theta_{2}(t, x)<0<\theta_{1}(t, x)$ for $(t, x) \in(N-$ int $F)$, $(t, x) \neq\left(t_{0}, x_{0}\right)$,

(ii) $L_{0}^{+} \theta_{1} \leq 0 \leq L_{0}^{+} \theta_{2}$ on $N$ - int $F$. Then $\left(t_{0}, x_{0}\right)$ is $Q^{+}$regular.

Proof. Let

$$
N_{r}=\left\{(t, x):\left|t-t_{0}\right|+\left\|x-x_{0}\right\| \leq r\right\} .
$$

For small enough $r, N_{r} \subset N$. We also let $M_{r}=N_{r} \cap\left(R^{m+1}-\right.$ int $\left.F\right)$. Write

$$
\begin{aligned}
& \delta_{1}(r)=\inf \left\{\theta_{1}(t, x):(t, x) \in \partial N_{r} \cap M_{r}\right\}, \\
& \delta_{2}(r)=\sup \left\{\theta_{2}(t, x):(t, x) \in \partial N_{r} \cap M_{r}\right\} .
\end{aligned}
$$

By a simple compactness argument $\delta_{1}(r)>0>\delta_{2}(r)$ for $r>0$. Write $O_{r}$ 
$=\left\{(t, x) \in N: \frac{1}{2} \delta_{1}(r)>\theta_{1}(t, x) \geq \theta_{2}(t, x)>\frac{1}{2} \delta_{2}(r)\right\}$; then $O_{r}$ is a neighborhood of $\left(t_{0}, x_{0}\right)$. Finally let

$$
\sup |h(t, x, y, z)|=H<\infty
$$

(for we may assume $N$ is bounded), and let $\lambda$ be the function of $r$ such that

$$
\lambda(r)=\sup _{(t, x) \in N_{r}}\left|g(t, x)-g\left(t_{0}, x_{0}\right)\right| .
$$

Now let $\varphi(t, x, y, z)=\partial \theta_{1} / \partial r+\nabla \theta_{1} \cdot f$ (we may suppose $\theta_{1}$ is defined on $R^{m+1}$ ). Taking $O_{r} \cap M_{r}$ as $B$ we apply Theorem 5.1; let $\eta$ be the function determined as in Theorem 5.1 satisfying (32). Suppose $(\bar{t}, \bar{x}) \in O_{r} \cap M_{r}$. Then there exists $\beta \in \Delta_{i}(s)$ such that for $\beta^{\prime} \in \Delta_{i}^{s}(\beta)$ and $y \in M_{1}(E)$, with $\xi(t)$ the trajectory determined by $\left(y, \beta^{\prime} y\right)$ :

$$
\varphi\left(t, \xi(t), y(t), \beta^{\prime} y(t)\right) \leq \Phi(t, \xi(t))+\eta(s) \text { for } \bar{t}+s \leq t \leq T .
$$

Here $\Phi(t, x)=\min _{z} \max _{y} \varphi(t, x, y, z)=L_{0}^{+} \theta_{1}(t, x)$, so $\varphi\left(t, \xi(t), y(t), \beta^{\prime} y(t)\right) \leq \eta(s)$ for $(t, \xi(t)) \in N$ and $\bar{t}+s \leq t \leq T$.

Suppose $B_{R}$ is a set large enough to contain every trajectory starting in $N$, and let

$$
A=\sup _{B_{R} \times Y \times z} \varphi(t, x, y, z) .
$$

With $\xi(t)$ the trajectory above, let $t^{\prime}$ be the time when $\xi(t)$ hits $\partial M_{r}$. Then $\left(t^{\prime}, \xi\left(t^{\prime}\right)\right)$ either belongs to $\partial N_{r}$ or to $\partial F$.

Suppose $\left(t^{\prime}, \xi\left(t^{\prime}\right)\right) \in \partial N_{r}$. Then $\theta_{1}\left(t^{\prime}, \xi\left(t^{\prime}\right)\right) \geq \delta_{1}(r) \geq \theta_{1}(\bar{t}, \xi(\bar{t}))+\frac{1}{2} \delta_{1}(r)$. Hence

$$
\int_{i}^{t^{\prime}}\left(\frac{\partial \theta_{1}}{\partial t}+\nabla \theta_{1} \cdot f\right) d t=\int_{i}^{t^{\prime}} \frac{d \theta_{1}}{d t} d t \geq \frac{1}{2} \delta_{1}(r)
$$

that is

$$
\int_{i}^{t^{\prime}} \varphi\left(t, \xi(t), y(t), \beta^{\prime} y(t)\right) d t \geq \frac{1}{2} \delta_{1}(r) .
$$

Therefore $\left(t^{\prime}-\bar{t}\right) \eta(s)+A s \geq \frac{1}{2} \delta_{1}(r)$, and, as $t^{\prime}-\bar{t} \leq 2 r$,

$$
2 r \eta(s)+A s \leq \frac{1}{2} \delta_{1}(r) .
$$

However, $\lim _{s \rightarrow 0} \eta(s)=0$ so $s$ can be chosen small enough so that $2 r \eta(s)+A s$ $<\frac{1}{2} \delta_{1}(r)$ thus contradicting (64). Therefore, $\left(t^{\prime}, \xi\left(t^{\prime}\right)\right) \in \partial F$ and $t^{\prime}=t_{F}$. Then

$$
\begin{aligned}
P\left(v, \beta^{\prime} y\right) & =\int_{i}^{t_{r}} h\left(t, \xi(t), y(t), \beta^{\prime} y(t)\right) d t+g\left(t_{F}, \xi\left(t_{F}\right)\right) \\
& \leq 2 H r+\lambda(r)+g\left(t_{0}, x_{0}\right)
\end{aligned}
$$

so that $v\left(\beta^{\prime}\right) \leq 2 H r+\lambda(r)+g\left(t_{0}, x_{0}\right)$. Hence $Q_{s}^{+}(\bar{t}, \bar{x}) \leq 2 H r+\lambda(r)+g\left(t_{0}, x_{0}\right)$ 
and so $\bar{Q}_{s}^{+}\left(t_{0}, x_{0}\right) \leq 2 H r+\lambda(r)+g\left(t_{0}, x_{0}\right)$ for $s$ small enough.

Therefore, since $\lim _{r \rightarrow 0} \lambda(r)=0$ we conclude that

$$
\lim _{s \rightarrow 0} \bar{Q}_{s}^{+}\left(t_{0}, x_{0}\right)=g\left(t_{0}, x_{0}\right) \text {. }
$$

A similar argument enables us to conclude that

$$
\lim _{(t, x) \rightarrow\left(t_{0}, x_{0}\right)} U(t, x)=g\left(t_{0}, x_{0}\right)
$$

and hence that $\left(t_{0}, x_{0}\right)$ is $Q^{+}$-regular.

Corollary 7.3. Suppose under the hypotheses of Theorem 7.2 we have, instead of $L_{0}^{+} \theta_{2} \geq 0$, that $L_{0}^{-} \theta_{2} \geq 0$ on $N$. Then $\left(t_{0}, x_{0}\right)$ is both $Q^{+}$- and $Q^{-}$-regular.

Proof. $L_{0}^{+} \theta_{2} \geq L_{0}^{-} \theta_{2}$ and $L_{0}^{+} \geq L_{0}^{-}$in general. Hence, by Theorem 7.2, $\left(t_{0}, x_{0}\right)$ is $Q^{+}$-regular. However, conversely we always have $L_{0}^{-} \theta_{1} \leq L_{0}^{+} \theta_{1} \leq 0$, and so we may apply the 'negative version' of Theorem 7.2 to deduce that $\left(t_{0}, x_{0}\right)$ is $Q^{-}$. regular.

The conditions of the corollary may be considered as allowing both players to force $(t, x(t))$ nearer $\left(t_{0}, x_{0}\right)$ from any initial position $\left(t_{1}, x_{1}\right)$. They are, therefore, a localized version of Friedman's conditions $(F)$ (see [4, p. 81]). We now prove that a generalization of Friedman's conditions ensures regularity.

Theorem 7.4. Suppose there exist $C^{1}$-functions $\theta_{1}$ and $\theta_{2}$ such that $\theta_{1}=\theta_{2}=0$ on $\partial F, \theta_{1} \geq 0 \geq \theta_{2}$ on $R^{m+1}-F$ and $L_{0}^{+} \theta_{1}<0<L_{0}^{+} \theta_{2}$ on $\partial F$. Then $\partial F$ is $Q^{+}$. regular.

Proof. For $\left(t_{0}, x_{0}\right) \in \partial F$ let

$$
\theta_{1}^{*}(t, x)=\theta_{1}(t, x)+\gamma_{1}\left(\left|t-t_{0}\right|^{2}+\left\|x-x_{0}\right\|^{2}\right) .
$$

Then for suitable $\gamma_{1}$ and in a small enough neighborhood of $\left(t_{0}, x_{0}\right)$

$$
L_{0}^{+} \theta_{1}^{*} \leq 0, \quad \theta_{1}^{*}>0,(t, x) \neq\left(t_{0}, x_{0}\right) .
$$

Similarly we construct

$$
\theta_{2}^{*}(t, x)=\theta_{2}(t, x)-\gamma_{2}\left(\left|t-t_{0}\right|^{2}+\left\|x-x_{0}\right\|^{2}\right)
$$

with small enough $\gamma_{2}$ so that in some neighborhood of $\left(t_{0}, x_{0}\right)$

$$
L_{0}^{+} \theta_{2}^{*} \geq 0, \quad \theta_{2}^{*}<0,(t, x) \neq\left(t_{0}, x_{0}\right) .
$$

Therefore, we may apply Theorem 7.2 to deduce that $\left(t_{0}, x_{0}\right)$ is $Q^{+}$-regular.

Friedman [4] uses $\rho$ and $-\rho$ for $\theta_{1}$ and $\theta_{2}$ and assumes $F$ is sufficiently smooth to guarantee the differentiability of $\rho$ near $\partial F$.

Of course, it is possible to 'mix' the criteria of Theorems 7.1 and 7.2. For example, for every point of $\partial F$ to be $Q^{+}$-regular it is sufficient that there exist $\theta_{1}$ as in Theorem 7.1 and for each $\left(t_{0}, x_{0}\right) \in \partial F$ a function $\theta_{2}$ as in Theorem 7.2. 
In the particular case of a generalized pursuit-evasion game where $g \equiv 0$ and $h \geq 0$, it is easy to see one has $L^{+}\left(\theta_{2}\right) \geq 0$ where $\theta_{2}$ is the function identically zero. Thus the existence of a function $\theta_{2}$ satisfying the conditions of Theorem 7.1 is automatically guaranteed by the type of game under consideration, and one only has to establish 'one-sided' conditions of the type of Theorems 7.2 or 7.4 to prove regularity.

8. Existence of value. Suppose now that $G$ is a game of prescribed duration (i.e. $\left.F=[T, \infty) \times R^{m}\right)$. Then if $G$ satisfies the Isaacs condition (see [2]):

$$
\min _{z} \max _{y}(p \cdot f+h)=\max _{y} \min _{z}(p \cdot f+h),
$$

for $t \leq T$ and $(x, p) \in R^{m} \times R^{m}$, we can show that $V^{+} \equiv V^{-}$(the main result of [2]). In this section we extend this result to games with regular boundary.

Theorem 8.1. Suppose the Isaacs condition (65) and the Lipschitz condition (43) are satisfied. Suppose further that $\partial F$ is $Q^{+}$- and $Q^{-}$-regular. Then $Q^{+} \equiv Q^{-}$.

Proof. By Theorem 6.3 and Corollary 3.4 we have for any nonanticipating stopping time $\tau$ :

$$
Q_{\tau}^{+}\left(t_{0}, x_{0} ; Q^{+}\right) \geq Q^{+}\left(t_{0}, x_{0}\right) \text { for }\left(t_{0}, x_{0}\right) \in R^{m+1}-\text { int } F .
$$

Similarly

$$
Q_{\tau}^{-}\left(t_{0}, x_{0} ; Q^{-}\right) \leq Q^{-}\left(t_{0}, x_{0}\right) .
$$

Now suppose $\tau$ is a constant such that $\tau \leq t_{F}$ for any possible trajectory. Then

$$
Q_{\tau}^{-}\left(t_{0}, x_{0} ; Q^{-}\right)=Q_{\tau}^{+}\left(t_{0}, x_{0} ; Q^{-}\right)
$$

by Lemma 2.2 and the main theorem (9.2) of [2]. Note that $Q^{+}$and $Q^{-}$are continuous. Hence

$$
\begin{aligned}
Q^{+}\left(t_{0}, x_{0}\right)-Q^{-}\left(t_{0}, x_{0}\right) & \leq Q_{\tau}^{+}\left(t_{0}, x_{0} ; Q^{+}\right)-Q_{\tau}^{-}\left(t_{0}, x_{0} ; Q^{-}\right) \\
& =Q_{\tau}^{+}\left(t_{0}, x_{0} ; Q^{+}\right)-Q_{\tau}^{+}\left(t_{0}, x_{0} ; Q^{-}\right) .
\end{aligned}
$$

We can now apply the inductive construction of Theorem 6.4 to deduce a contradiction if $Q^{+}\left(t_{0}, x_{0}\right) \neq Q^{-}\left(t_{0}, x_{0}\right)$. Therefore, $Q^{+}\left(t_{0}, x_{0}\right)=Q^{-}\left(t_{0}, x_{0}\right)$ for all $\left(t_{0}, x_{0}\right) \in R^{m+1}-$ int $F$.

It is worth observing that the Isaacs condition (65) implies $L^{+} \equiv L^{-}$and also that $L_{0}^{+} \equiv L_{0}^{-}$. Hence, the sufficient conditions for $Q^{+}$-regularity of $\$ 7$ will also ensure $Q^{-}$-regularity. It seems reasonable to conjecture that $Q^{+}$- and $Q^{-}$regularity are equivalent in general if the Isaacs condition is satisfied.

\section{REFERENCES}

1. R. J. Elliott and N. J. Kalton, Values in differential games, Bull. Amer. Math. Soc. 78 (1972), 427-431. MR 45 \#4840. 
2.—, The existence of value in differential games, Mem. Amer. Math. Soc. No. 126 (1972).

3. - The existence of value in differential games of pursuit and evasion, J. Differential Equations 12 (1972), 504-523.

4. A. Friedman, Differential games, Pure and Appl. Math., vol. 25, Wiley, New York and London, 1971.

5. - Comparison theorems for differential games. I, II, J. Differential Equations 12 (1972), I:162-172, II: 396-416.

6. P. R. Halmos, Measure theory, Van Nostrand, Princeton, N.J., 1950. MR 11, 504.

7. J. G. Hocking and G. S. Young, Topology, Addison-Wesley, Reading, Mass. and London, 1961. MR 23 \#A 2857.

8. R. Isaacs, Differential games, Wiley, New York, 1965. MR 35 \# 1362.

9. M. Krzyzański, Partial differential equations of second order. Vol. 1, Monografie Mat., Tom 53, PWN, Warsaw, 1957; English transl., PWN, Warsaw, 1971. MR 20 \#6576; MR 43 \#3597.

10. O. Perron, Eine neue Behandlung der ersten Randwertproblem für $\Delta u=0$, Math. Z. 18 (1923), 42-54.

11. A. N. V. Rao, Comparison of differential games of fixed duration, SIAM Control 10 (1972), 393-397.

DEPARTMENT OF PURE MATHEMATICS, UNIVERSITY OF HULL, 22 NEWLAND PARK, HULL, HUS 2DW, ENGLAND

DEPARTMENT OF PURE MATHEMATICS, UNIVERSITY COLLEGE, SWANSEA, WALES 